\title{
Analytical Model of Random Variation in Drain Current of FGMOSFET
}

\author{
Rawid Banchuin \\ Department of Computer Engineering, Siam University, 235 Petchakasem Road, Bangkok 10163, Thailand
}

Correspondence should be addressed to Rawid Banchuin; rawid_b@yahoo.com

Received 15 March 2015; Revised 4 June 2015; Accepted 11 June 2015

Academic Editor: Jiun-Wei Horng

Copyright (C) 2015 Rawid Banchuin. This is an open access article distributed under the Creative Commons Attribution License, which permits unrestricted use, distribution, and reproduction in any medium, provided the original work is properly cited.

The analytical model of random variation in drain current of the Floating Gate MOSFET (FGMOSFET) has been proposed in this research. The model is composed of two parts for triode and saturation region of operation where the process induced device level random variations of each region and their statistical correlations have been taken into account. The nonlinearity of floating gate voltage and dependency on drain voltage of the coupling factors of FGMOSFET have also been considered. The model has been found to be very accurate since it can accurately fit the SPICE BSIM3v3 based reference obtained by using Monte-Carlo SPICE simulation and FGMOSFET simulation technique with SPICE. It can fit the BSIM4 based reference if desired by using the optimally extracted parameters. By using the proposed model, the variability analysis of FGMOSFET and the analytical modeling of the variation in the circuit level parameter of any FGMOSFET based circuit can be performed. So, this model has been found to be an efficient tool for the variability aware analysis and design of FGMOSFET based circuit.

\section{Introduction}

FGMOSFETs have been extensively utilized in various ana$\log /$ digital circuits such as [1-9]. Similarly to the MOSFET based circuits, the performances of the FGMOSFET based circuits have been deteriorated by process induced device level random variations [10-12]. This is because these device level variations yield random variations in circuit level parameters, for example, drain current $\left(I_{D}\right)$ and transconductance. These variations yield variations in parameters of FGMOSFET based circuit such as transconductance of the FGMOSFET based voltage to current converter [1] and equivalent resistance of the FGMOSFET based voltage controlled resistor [2,3]. For handling this issue, variability aware analysis/design concept has been applied in the designing of many FGMOSFET based circuits, for example, [4-6].

Similarly to MOSFET, $I_{D}$ has been found to be the key circuit level parameter of FGMOSFET as it is directly measurable and can be the basis for determining the others. According to its importance, the analytical models of process induced random variation and mismatch in $I_{D}$ of MOSFET have been proposed without regard to certain circuit in many previous researches, for example, [13-15]. So, their results are applicable to any MOSFET based circuit. For the FGMOSFET, most of the previous work has been oriented to certain FGMOSFET circuits, for example, [16-19]. Some previous studies have not been devoted to any circuit, for example, $[20,21]$. However, the results of these works are incomplete since the statistical correlations between device level random variations have been neglected. Moreover, nonlinearity of floating gate voltage and dependency on drain voltage of the coupling factors, which are the important features of FGMOSFET [22], have not been considered.

So, the analytical model of process induced random variation in $I_{D}\left(\Delta I_{D}\right)$ of the FGMOSFET has been proposed in this research. The proposed model is composed of two parts for triode and saturation regions of operation where the process induced device level random variations of each region and their statistical correlations have been taken into account. The nonlinearity of floating gate voltage and dependency on drain voltage of the coupling factors of FGMOSFET have also been considered. This model has been formulated without regard to any circuit. So, it is applicable to all FGMOSFET based circuits. It has been found to be very accurate since it can fit the SPICE BSIM3v3 based reference obtained by using FGMOSFET simulation technique with SPICE [23] and 


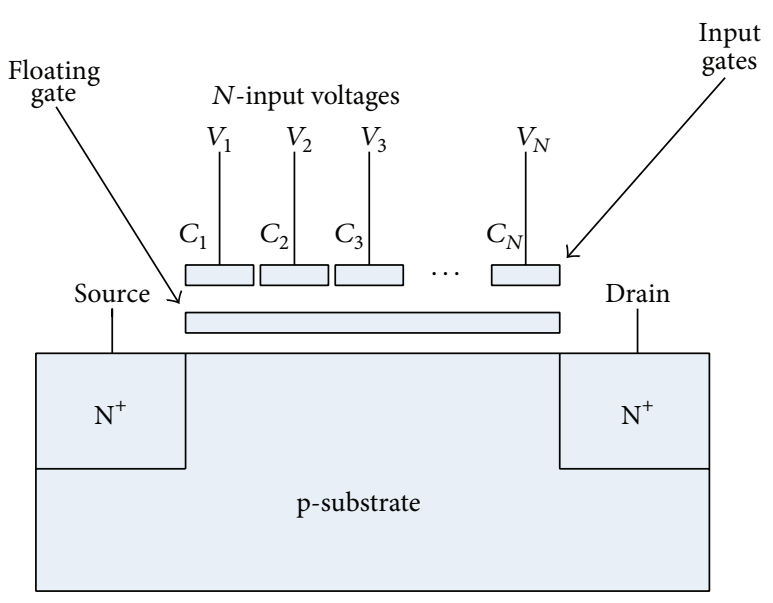

FIGURE 1: A cross-sectional view of N-type $N$ inputs FGMOSFET [2].

Monte-Carlo SPICE simulation with very high accuracy. If desired, it can fit the BSIM4 based reference by applying the optimum parameters extracted by using the optimization algorithm [24]. The variability analysis of FGMOSFET and the analytical modeling of process induced random variation in circuit level parameter of any FGMOSFET based circuit can be performed by using the proposed model as the basis. Hence, this model has been found to be an efficient tool for the variability aware analysis/design of any circuit involving FGMOSFET.

\section{The Overview of FGMOSFET}

FGMOSFET is a special type of MOSFET with an additional gate isolated within the oxide, namely, the floating gate [2]. A cross-sectional view of an N-type FGMOSFET with $N$ inputs implemented as $N$ discrete input gates where $N>1$ can be shown in Figure 1.

It should be mentioned here that the dimension (width and length) of any input gate determines the magnitude of its corresponding input capacitance. The symbol and equivalent circuit of $N$ inputs FGMOSFET are shown in Figure 2. Such equivalent circuit is composed of a MOSFET, $N$ input capacitances $\left(C_{1}, C_{2}, C_{3}, \ldots, C_{N}\right)$, overlap capacitance between floating gate and drain $\left(C_{\mathrm{fd}}\right)$, overlap capacitance between floating gate and source $\left(C_{\mathrm{fs}}\right)$, and parasitic capacitance between floating gate and substrate $\left(C_{\mathrm{fb}}\right)$ [2].

Let $\{i\}=\{1,2,3, \ldots, N\}$ and let any $i$ th input capacitance be denoted by $C_{i}$; the floating gate voltage, $V_{\mathrm{FG}}$, can be given by [2]

$$
V_{\mathrm{FG}}=\frac{\sum_{i=1}^{N}\left[C_{i} V_{i}\right]+C_{\mathrm{fd}} V_{D}+C_{\mathrm{fs}} V_{S}+C_{\mathrm{fb}} V_{B}}{C_{T}}
$$

where $V_{i}$ is the input voltage at any ith input gate, $V_{D}$ is the drain voltage, $V_{S}$ is the source voltage, and $V_{B}$ is the bulk voltage. Moreover, $C_{T}$ denotes the total capacitance of the floating gate which can be defined as [2]

$$
C_{T}=\sum_{i=1}^{N}\left[C_{i}\right]+C_{\mathrm{fd}}+C_{\mathrm{fs}}+C_{\mathrm{fb}} .
$$

Let $k_{i}, k_{\mathrm{fd}}, k_{\mathrm{fs}}$, and $k_{\mathrm{fb}}$ denote the coupling factor of any $i$ th input gate, drain, source, and bulk and let them be defined as $k_{i}=C_{i} / C_{T}, k_{\mathrm{fd}}=C_{\mathrm{fd}} / C_{T}, k_{\mathrm{fs}}=C_{\mathrm{fs}} / C_{T}$, and $k_{\mathrm{fb}}=C_{\mathrm{fb}} / C_{T}$, respectively; $V_{\mathrm{FG}}$ can be alternatively given as follows:

$$
V_{\mathrm{FG}}=\sum_{i=1}^{N}\left[k_{i} V_{i}\right]+k_{\mathrm{fd}} V_{D}+k_{\mathrm{fs}} V_{S}+k_{\mathrm{fb}} V_{B}
$$

From either (1) or (3), it can be seen that $V_{\mathrm{FG}}$ depends on $V_{i}, V_{D}, V_{S}$, and $V_{B}$. According to [22], $V_{\mathrm{FG}}$ of FGMOSFET in triode is a nonlinear function of $V_{D}$ and $C_{T}$ depends on $V_{D}$ as $C_{\mathrm{fb}}$ does. So, $k_{i}, k_{\mathrm{fd}}, k_{\mathrm{fs}}$, and $k_{\mathrm{fb}}$ are dependent on $V_{D}$ since they are functions of $C_{T}$. For FGMOSFET in saturation region, $C_{\mathrm{fb}}$ is constant and so are $C_{T}, k_{i}, k_{\mathrm{fd}}, k_{\mathrm{fs}}$, and $k_{\mathrm{fb}}$. As a result, $V_{\mathrm{FG}}$ become a linear function of $V_{D}$.

\section{Formulation of the Proposed Model}

3.1. Triode Region Part. Firstly, $I_{D}$ of FGMOSFET in triode region will be formulated by letting the gate to source voltage $\left(V_{\mathrm{GS}}\right)$ in the analytical model of $I_{D}$ of MOSFET in such region be replaced by $V_{\mathrm{FG}}-V_{S}$. The second order effects such as mobility degradation and short channel effect have been taken into account for making the model applicable to deeply scaled technology. In order to do so, the linear model of mobility degradation [15] has been adopted. Hence, $I_{D}$ of the MOSFET in triode can be given by

$$
\begin{aligned}
& I_{D} \\
& \quad=\beta\left[1-\theta\left(V_{\mathrm{GS}}-V_{\mathrm{TH}}\right)\right]\left[\left(V_{\mathrm{GS}}-V_{\mathrm{TH}}\right) V_{\mathrm{DS}}-\frac{1}{2} V_{\mathrm{DS}}^{2}\right],
\end{aligned}
$$

where $V_{\mathrm{DS}}, V_{\mathrm{TH}}, \beta$, and $\theta$ denote drain to source voltage, threshold voltage, current factor, and mobility degradation coefficient, respectively.

Since $V_{\mathrm{FG}}$ of FGMOSFET in this region is a nonlinear function of $V_{D}, V_{\mathrm{FG}}$ can be given by

$$
\begin{aligned}
V_{\mathrm{FG}}= & \sum_{i=1}^{N}\left[k_{i}\left(V_{D}\right) V_{i}\right]+k_{\mathrm{fd}}\left(V_{D}\right) V_{D}+k_{\mathrm{fs}}\left(V_{D}\right) V_{S} \\
& +k_{\mathrm{fb}}\left(V_{D}\right) V_{B},
\end{aligned}
$$




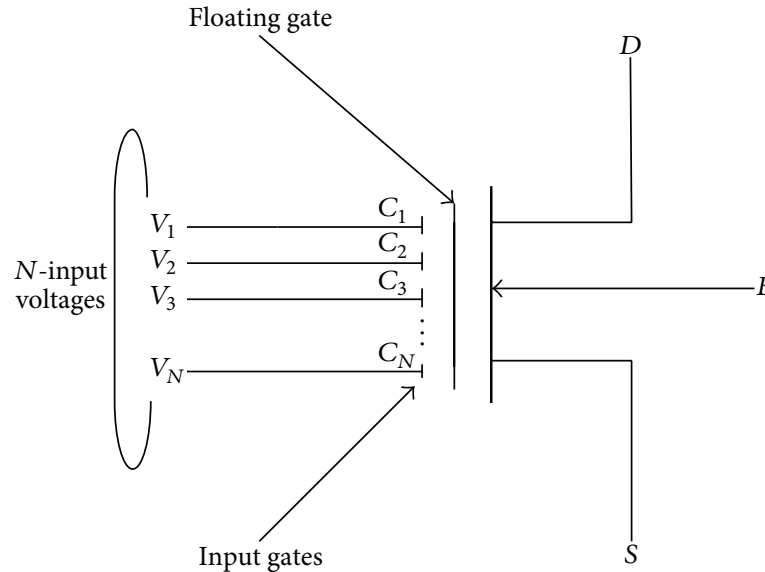

(a)

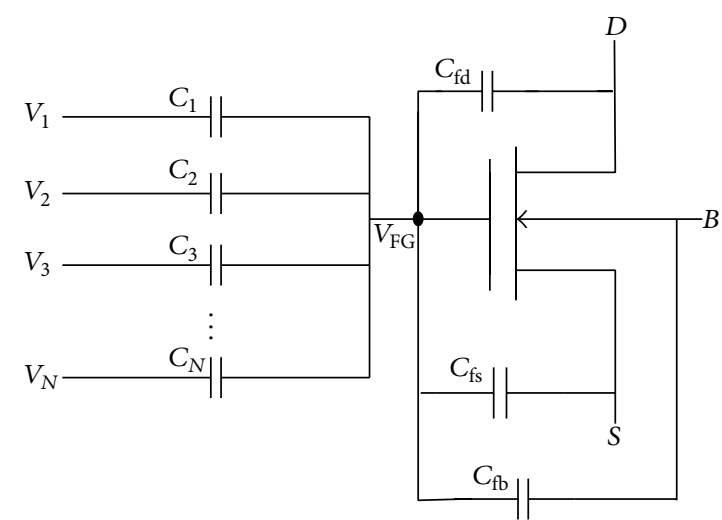

(b)

FIGURE 2: The symbol (a) and equivalent circuit model (b) of N-type $N$ inputs FGMOSFET [2].

where $k_{i}\left(V_{D}\right), k_{\mathrm{fd}}\left(V_{D}\right), k_{\mathrm{fs}}\left(V_{D}\right)$, and $k_{\mathrm{fb}}\left(V_{D}\right)$, which are, respectively, $V_{D}$ dependent $k_{i}, k_{\mathrm{fd}}, k_{\mathrm{fs}}$, and $k_{\mathrm{fb}}$, can be modeled as power series of $V_{D}$ as follows:

$$
\begin{aligned}
k_{i}\left(V_{D}\right) & =\sum_{m=0}^{\infty}\left[\alpha_{i m} V_{D}^{m}\right], \\
k_{\mathrm{fd}}\left(V_{D}\right) & =\sum_{m=0}^{\infty}\left[\alpha_{d m} V_{D}^{m}\right], \\
k_{\mathrm{fs}}\left(V_{D}\right) & =\sum_{m=0}^{\infty}\left[\alpha_{s m} V_{D}^{m}\right], \\
k_{\mathrm{fb}}\left(V_{D}\right) & =\sum_{m=0}^{\infty}\left[\alpha_{b m} V_{D}^{m}\right],
\end{aligned}
$$

where $\alpha_{i m}, \alpha_{d m}, \alpha_{s m}$, and $\alpha_{b m}$ are coefficients of the power series representation of $k_{i}\left(V_{D}\right), k_{\mathrm{fd}}\left(V_{D}\right), k_{\mathrm{fs}}\left(V_{D}\right)$, and $k_{\mathrm{fb}}\left(V_{D}\right)$, respectively.

As $V_{D}$ can be very low in recent CMOS technology, high order terms of $k_{i}\left(V_{D}\right), k_{\mathrm{fd}}\left(V_{D}\right), k_{\mathrm{fs}}\left(V_{D}\right)$, and $k_{\mathrm{fb}}\left(V_{D}\right)$ can be neglected. So, (6)-(9) become (11)-(13) where $\alpha_{i 0}, \alpha_{d 0}, \alpha_{s 0}$, and $\alpha_{b 0}$ and $\alpha_{i 1}, \alpha_{d 1}, \alpha_{s 1}$, and $\alpha_{b 1}$ are $\alpha_{i m}, \alpha_{d m}, \alpha_{s m}$, and $\alpha_{b m}$ with $m=0$ and $m=1$ :

$$
\begin{gathered}
k_{i}\left(V_{D}\right)=\alpha_{i 0}+\alpha_{i 1} V_{D}, \\
k_{\mathrm{fd}}\left(V_{D}\right)=\alpha_{d 0}+\alpha_{d 1} V_{D}, \\
k_{\mathrm{fs}}\left(V_{D}\right)=\alpha_{s 0}+\alpha_{s 1} V_{D}, \\
k_{\mathrm{fb}}\left(V_{D}\right)=\alpha_{b 0}+\alpha_{b 1} V_{D} .
\end{gathered}
$$

By using (4), (10)-(13), and the methodology stated above, $I_{D}$ of the triode region operated FGMOSFET is given by (14) as $\alpha_{s 0}+\alpha_{s 1} V_{D} \ll 1$ :

$$
\begin{aligned}
I_{D} & =\beta\left[1-\theta\left(\sum_{i=1}^{N}\left[\left(\alpha_{i 0}+\alpha_{i 1} V_{D}\right) V_{i}\right]\right.\right. \\
& +\left(\alpha_{d 0}+\alpha_{d 1} V_{D}\right) V_{D}+\left(\alpha_{b 0}+\alpha_{b 1} V_{D}\right) V_{D}-V_{S} \\
& \left.\left.-V_{\mathrm{TH}}\right)\right]\left[\left(\sum_{i=1}^{N}\left[\left(\alpha_{i 0}+\alpha_{i 1} V_{D}\right) V_{i}\right]\right.\right. \\
& +\left(\alpha_{d 0}+\alpha_{d 1} V_{D}\right) V_{D}+\left(\alpha_{b 0}+\alpha_{b 1} V_{D}\right) V_{D}-V_{S} \\
& \left.\left.-V_{\mathrm{TH}}\right) V_{\mathrm{DS}}-\frac{1}{2} V_{\mathrm{DS}}^{2}\right] .
\end{aligned}
$$

It can be seen from (14) that the process induced device level random variations of FGMOSFET in triode are random variations in $V_{\mathrm{TH}}, \alpha_{i 0}, \alpha_{i 1}, \alpha_{d 0}, \alpha_{d 1}, \alpha_{b 0}, \alpha_{b 1}, \beta$, and $\theta$ denoted by $\Delta V_{\mathrm{TH}}, \Delta \alpha_{i 0}, \Delta \alpha_{i 1}, \Delta \alpha_{d 0}, \Delta \alpha_{d 1}, \Delta \alpha_{b 0}, \Delta \alpha_{b 1}, \Delta \beta$, and $\Delta \theta$, respectively. So, $\Delta I_{D}$ of FGMOSET in triode region can be given by (15) where all derivatives given by (16)-(24) can be found by using (14):

$$
\begin{aligned}
\Delta I_{D} & =\left(\frac{\partial I_{D}}{\partial V_{\mathrm{TH}}}\right) \Delta V_{\mathrm{TH}}+\left(\frac{\partial I_{D}}{\partial \beta}\right) \Delta \beta+\left(\frac{\partial I_{D}}{\partial \theta}\right) \Delta \theta \\
& +\left(\frac{\partial I_{D}}{\partial \alpha_{d 0}}\right) \Delta \alpha_{d 0}+\left(\frac{\partial I_{D}}{\partial \alpha_{d 1}}\right) \Delta \alpha_{d 1}+\left(\frac{\partial I_{D}}{\partial \alpha_{b 0}}\right) \Delta \alpha_{b 0} \\
& +\left(\frac{\partial I_{D}}{\partial \alpha_{b 1}}\right) \Delta \alpha_{b 1}+\sum_{i=1}^{N}\left[\left(\frac{\partial I_{D}}{\partial \alpha_{i 0}}\right) \Delta \alpha_{i 0}\right] \\
& +\sum_{i=1}^{N}\left[\left(\frac{\partial I_{D}}{\partial \alpha_{i 1}}\right) \Delta \alpha_{i 1}\right],
\end{aligned}
$$




$$
\begin{aligned}
& \frac{\partial I_{D}}{\partial V_{\mathrm{TH}}}=\frac{\beta}{2} V_{\mathrm{DS}}\left[4 \theta \left(\alpha_{b 1} V_{B} V_{D}+\alpha_{b 0} V_{B}\right.\right. \\
& \left.+\left(\alpha_{d 0}+\alpha_{d 1} V_{D}\right) V_{D}+\sum_{i=1}^{N}\left[\left(\alpha_{i 0}+\alpha_{i 1} V_{D}\right) V_{i}\right]\right) \\
& \left.-\theta\left(4\left(V_{S}+V_{\mathrm{TH}}\right)+V_{\mathrm{DS}}\right)-2\right], \\
& \frac{\partial I_{D}}{\partial \beta}=\left[1-\theta\left(\sum_{i=1}^{N}\left[\left(\alpha_{i 0}+\alpha_{i 1} V_{D}\right) V_{i}\right]\right.\right. \\
& +\left(\alpha_{d 0}+\alpha_{d 1} V_{D}\right) V_{D}+\left(\alpha_{b 0}+\alpha_{b 1} V_{D}\right) V_{D}-V_{S} \\
& \left.\left.-V_{\mathrm{TH}}\right)\right]\left[\left(\sum_{i=1}^{N}\left[\left(\alpha_{i 0}+\alpha_{i 1} V_{D}\right) V_{i}\right]\right.\right. \\
& +\left(\alpha_{d 0}+\alpha_{d 1} V_{D}\right) V_{D}+\left(\alpha_{b 0}+\alpha_{b 1} V_{D}\right) V_{D}-V_{S} \\
& \left.\left.-V_{\mathrm{TH}}\right) V_{\mathrm{DS}}-\frac{1}{2} V_{\mathrm{DS}}^{2}\right] \text {, } \\
& \frac{\partial I_{D}}{\partial \theta}=\beta\left[V_{S}+V_{\mathrm{TH}}-V_{B}\left(\alpha_{b 0}+\alpha_{b 1} V_{D}\right)-V_{D}\left(\alpha_{d 0}\right.\right. \\
& \left.\left.+\alpha_{d 1} V_{D}\right)-\sum_{i=1}^{N}\left[\left(\alpha_{i 0}+\alpha_{i 1} V_{D}\right) V_{i}\right]\right]-\frac{1}{2} V_{\mathrm{DS}}\left(V_{\mathrm{DS}}\right. \\
& \left.+2\left(V_{S}+V_{\mathrm{TH}}\right)\right)+V_{\mathrm{DS}}\left(\alpha_{b 1} V_{B} V_{D}+\alpha_{b 0} V_{B}+\left(\alpha_{d 0}\right.\right. \\
& \left.\left.+\alpha_{d 1} V_{D}\right) V_{D}+\sum_{i=1}^{N}\left[\left(\alpha_{i 0}+\alpha_{i 1} V_{D}\right) V_{i}\right]\right) \text {, } \\
& \frac{\partial I_{D}}{\partial \alpha_{d 0}}=\frac{\beta}{2} V_{D} V_{\mathrm{DS}}\left[2+\theta V_{\mathrm{DS}}+4 \theta\left(V_{S}+V_{\mathrm{TH}}\right)\right. \\
& -4 \theta\left(\alpha_{b 1} V_{B} V_{D}+\alpha_{b 0} V_{B}+\left(\alpha_{d 0}+\alpha_{d 1} V_{D}\right) V_{D}\right. \\
& \left.\left.+\sum_{i=1}^{N}\left[\left(\alpha_{i 0}+\alpha_{i 1} V_{D}\right) V_{i}\right]\right)\right] \\
& \frac{\partial I_{D}}{\partial \alpha_{b 0}}=\frac{\beta}{2} V_{B} V_{\mathrm{DS}}\left[2+\theta V_{\mathrm{DS}}+4 \theta\left(V_{S}+V_{\mathrm{TH}}\right)\right. \\
& -4 \theta\left(\alpha_{b 1} V_{B} V_{D}+\alpha_{b 0} V_{B}+\left(\alpha_{d 0}+\alpha_{d 1} V_{D}\right) V_{D}\right. \\
& \left.\left.+\sum_{i=1}^{N}\left[\left(\alpha_{i 0}+\alpha_{i 1} V_{D}\right) V_{i}\right]\right)\right]
\end{aligned}
$$

$$
\begin{aligned}
& \frac{\partial I_{D}}{\partial \alpha_{d 1}}=\frac{\beta}{2} V_{\mathrm{DS}} V_{D}^{2}\left[2+\theta V_{\mathrm{DS}}+4 \theta\left(V_{S}+V_{\mathrm{TH}}\right)\right. \\
& -4 \theta\left(\alpha_{b 1} V_{B} V_{D}+\alpha_{b 0} V_{B}+\left(\alpha_{d 0}+\alpha_{d 1} V_{D}\right) V_{D}\right. \\
& \left.\left.+\sum_{i=1}^{N}\left[\left(\alpha_{i 0}+\alpha_{i 1} V_{D}\right) V_{i}\right]\right)\right] \text {, } \\
& \frac{\partial I_{D}}{\partial \alpha_{b 1}}=\frac{\beta}{2} V_{B} V_{D} V_{\mathrm{DS}}\left[2+\theta V_{\mathrm{DS}}+4 \theta\left(V_{S}+V_{\mathrm{TH}}\right)\right. \\
& -4 \theta\left(\alpha_{b 1} V_{B} V_{D}+\alpha_{b 0} V_{B}+\left(\alpha_{d 0}+\alpha_{d 1} V_{D}\right) V_{D}\right. \\
& \left.\left.+\sum_{i=1}^{N}\left[\left(\alpha_{i 0}+\alpha_{i 1} V_{D}\right) V_{i}\right]\right)\right] \\
& \frac{\partial I_{D}}{\partial \alpha_{i 0}}=\frac{\beta}{2} V_{\mathrm{DS}} \sum_{i=1}^{N}\left[V_{i}\right]\left[2+\theta V_{\mathrm{DS}}+4 \theta\left(V_{\mathrm{S}}+V_{\mathrm{TH}}\right)\right. \\
& -4 \theta\left(\alpha_{b 1} V_{B} V_{D}+\alpha_{b 0} V_{B}+\left(\alpha_{d 0}+\alpha_{d 1} V_{D}\right) V_{D}\right. \\
& \left.\left.+\sum_{i=1}^{N}\left[\left(\alpha_{i 0}+\alpha_{i 1} V_{D}\right) V_{i}\right]\right)\right] \\
& \frac{\partial I_{D}}{\partial \alpha_{i}}=\frac{\beta}{2} V_{D} V_{\mathrm{DS}} \sum_{i=1}^{N}\left[V_{i}\right]\left[2+\theta V_{\mathrm{DS}}+4 \theta\left(V_{S}+V_{\mathrm{TH}}\right)\right. \\
& -4 \theta\left(\alpha_{b 1} V_{B} V_{D}+\alpha_{b 0} V_{B}+\left(\alpha_{d 0}+\alpha_{d 1} V_{D}\right) V_{D}\right. \\
& \left.\left.+\sum_{i=1}^{N}\left[\left(\alpha_{i 0}+\alpha_{i 1} V_{D}\right) V_{i}\right]\right)\right]
\end{aligned}
$$

Since $\Delta V_{\mathrm{TH}}, \Delta \alpha_{i 0}, \Delta \alpha_{i 1}, \Delta \alpha_{d 0}, \Delta \alpha_{d 1}, \Delta \alpha_{b 0}, \Delta \alpha_{b 1}, \Delta \beta$, and $\Delta \theta$ are random variables, so are $\Delta I_{D}$ and the statistical behavior of $\Delta I_{D}$ must be analytically modeled for its complete modeling. In order to do so, its average $\left(\Delta I_{D \text {, avr }}\right)$ and variance $\left(\sigma_{\Delta I_{D}}^{2}\right)$ must be formulated. As a result, $\Delta I_{D \text {,avr }}=0$ similar to those of $\Delta V_{\mathrm{TH}}, \Delta \alpha_{i 0}, \Delta \alpha_{i 1}, \Delta \alpha_{d 0}, \Delta \alpha_{d 1}, \Delta \alpha_{b 0}, \Delta \alpha_{b 1}, \Delta \beta$, and $\Delta \theta$ and $\sigma_{\Delta I_{D}}^{2}$ of FGMOSFET in triode can be given by taking the statistical correlations of $\Delta V_{\mathrm{TH}}, \Delta \alpha_{i 0}, \Delta \alpha_{i 1}, \Delta \alpha_{d 0}, \Delta \alpha_{d 1}, \Delta \alpha_{b 0}$, $\Delta \alpha_{b 1}, \Delta \beta$, and $\Delta \theta$ into account as follows:

$$
\begin{gathered}
\sigma_{\Delta I_{D}}^{2}=\left(\frac{\partial I_{D}}{\partial V_{\mathrm{TH}}}\right)^{2} \sigma_{\Delta V_{\mathrm{TH}}}^{2}+\left(\frac{\partial I_{D}}{\partial \beta}\right)^{2} \sigma_{\Delta \beta}^{2}+\left(\frac{\partial I_{D}}{\partial \theta}\right)^{2} \sigma_{\Delta \theta}^{2} \\
+\left(\frac{\partial I_{D}}{\partial \alpha_{d 0}}\right)^{2} \sigma_{\Delta \alpha_{d 0}}^{2}+\left(\frac{\partial I_{D}}{\partial \alpha_{d 1}}\right)^{2} \sigma_{\Delta \alpha_{d 1}}^{2}+\left(\frac{\partial I_{D}}{\partial \alpha_{b 0}}\right)^{2} \\
\cdot \sigma_{\Delta \alpha_{b 0}}^{2}+\left(\frac{\partial I_{D}}{\partial \alpha_{b 1}}\right)^{2} \sigma_{\Delta \alpha_{b 1}}^{2}+\sum_{i=1}^{N}\left[\left(\frac{\partial I_{D}}{\partial \alpha_{i 0}}\right)^{2} \sigma_{\Delta \alpha_{i 0}}^{2}\right]
\end{gathered}
$$


Active and Passive Electronic Components

5

$$
\begin{aligned}
& +\sum_{i=1}^{N}\left[\left(\frac{\partial I_{D}}{\partial \alpha_{i 1}}\right)^{2} \sigma_{\Delta \alpha_{i 1}}^{2}\right]+2\left(\frac{\partial I_{D}}{\partial V_{\mathrm{TH}}}\right)\left(\frac{\partial I_{D}}{\partial \beta}\right) \\
& \left.\cdot \rho_{\Delta \theta, \Delta \alpha_{i 0}} \sqrt{\sigma_{\Delta \theta}^{2}} \sqrt{\sigma_{\Delta \alpha_{i 0}}^{2}}\right]+2 \sum_{i=1}^{N}\left[\left(\frac{\partial I_{D}}{\partial \theta}\right)\left(\frac{\partial I_{D}}{\partial \alpha_{i 1}}\right)\right. \\
& \cdot \rho_{\Delta V_{\mathrm{TH}}, \Delta \beta} \sqrt{\sigma_{\Delta V_{\mathrm{TH}}^{2}}} \sqrt{\sigma_{\Delta \beta}^{2}}+2\left(\frac{\partial I_{D}}{\partial V_{\mathrm{TH}}}\right)\left(\frac{\partial I_{D}}{\partial \theta}\right) \\
& \left.\cdot \rho_{\Delta \theta, \Delta \alpha_{i 1}} \sqrt{\sigma_{\Delta \theta}^{2}} \sqrt{\sigma_{\Delta \alpha_{i 1}}^{2}}\right]+2\left(\frac{\partial I_{D}}{\partial \alpha_{d 0}}\right)\left(\frac{\partial I_{D}}{\partial \alpha_{d 1}}\right) \\
& \cdot \rho_{\Delta V_{\mathrm{TH}}, \Delta \theta} \sqrt{\sigma_{\Delta V_{\mathrm{TH}}^{2}}} \sqrt{\sigma_{\Delta \theta}^{2}}+2\left(\frac{\partial I_{D}}{\partial V_{\mathrm{TH}}}\right)\left(\frac{\partial I_{D}}{\partial \alpha_{d 0}}\right) \\
& \cdot \rho_{\Delta \alpha_{d 0}, \Delta \alpha_{d 1}} \sqrt{\sigma_{\Delta \alpha_{d 0}}^{2}} \sqrt{\sigma_{\Delta \alpha_{d 1}}^{2}}+2\left(\frac{\partial I_{D}}{\partial \alpha_{d 0}}\right)\left(\frac{\partial I_{D}}{\partial \alpha_{b 0}}\right) \\
& \cdot \rho_{\Delta V_{\mathrm{TH}}, \Delta \alpha_{d 0}} \sqrt{\sigma_{\Delta V_{\mathrm{TH}}}^{2}} \sqrt{\sigma_{\Delta \alpha_{d 0}}^{2}}+2\left(\frac{\partial I_{D}}{\partial V_{\mathrm{TH}}}\right)\left(\frac{\partial I_{D}}{\partial \alpha_{d 1}}\right) \\
& \cdot \rho_{\Delta \alpha_{d 0}, \Delta \alpha_{b 0}} \sqrt{\sigma_{\Delta \alpha_{d 0}}^{2}} \sqrt{\sigma_{\Delta \alpha_{b 0}}^{2}}+2\left(\frac{\partial I_{D}}{\partial \alpha_{d 0}}\right)\left(\frac{\partial I_{D}}{\partial \alpha_{b 1}}\right) \\
& \cdot \rho_{\Delta V_{\mathrm{TH}}, \Delta \theta} \sqrt{\sigma_{\Delta V_{\mathrm{TH}}}^{2}} \sqrt{\sigma_{\Delta \alpha_{d 1}}^{2}}+2\left(\frac{\partial I_{D}}{\partial V_{\mathrm{TH}}}\right)\left(\frac{\partial I_{D}}{\partial \alpha_{b 0}}\right) \\
& \cdot \rho_{\Delta V_{\mathrm{TH}}, \Delta \alpha_{d 0}} \sqrt{\sigma_{\Delta V_{\mathrm{TH}}}^{2}} \sqrt{\sigma_{\Delta \alpha_{b 0}}^{2}}+2\left(\frac{\partial I_{D}}{\partial V_{\mathrm{TH}}}\right)\left(\frac{\partial I_{D}}{\partial \alpha_{b 1}}\right) \\
& \cdot \rho_{\Delta \alpha_{d 0}, \Delta \alpha_{b 1}} \sqrt{\sigma_{\Delta \alpha_{d 0}}^{2}} \sqrt{\sigma_{\Delta \alpha_{b 1}}^{2}}+2 \sum_{i=1}^{N}\left[\left(\frac{\partial I_{D}}{\partial \alpha_{d 0}}\right)\left(\frac{\partial I_{D}}{\partial \alpha_{i 0}}\right)\right. \\
& \left.\cdot \rho_{\Delta \alpha_{d 0}, \Delta \alpha_{i 0}} \sqrt{\sigma_{\Delta \alpha_{d 0}}^{2}} \sqrt{\sigma_{\Delta \alpha_{i 0}}^{2}}\right]+2 \sum_{i=1}^{N}\left[\left(\frac{\partial I_{D}}{\partial \alpha_{d 0}}\right)\left(\frac{\partial I_{D}}{\partial \alpha_{i 1}}\right)\right. \\
& \cdot \rho_{\Delta V_{\mathrm{TH}}, \Delta \theta} \sqrt{\sigma_{\Delta V_{\mathrm{TH}}}^{2}} \sqrt{\sigma_{\Delta \alpha_{b 1}}^{2}}+2 \sum_{i=1}^{N}\left[\left(\frac{\partial I_{D}}{\partial V_{\mathrm{TH}}}\right)\left(\frac{\partial I_{D}}{\partial \alpha_{i 0}}\right)\right. \\
& \left.\cdot \rho_{\Delta V_{\mathrm{TH}}, \Delta \alpha_{i 0}} \sqrt{\sigma_{\Delta V_{\mathrm{TH}}^{2}}^{2}} \sqrt{\sigma_{\Delta \alpha_{i 0}}^{2}}\right]+2 \sum_{i=1}^{N}\left[\left(\frac{\partial I_{D}}{\partial V_{\mathrm{TH}}}\right)\right. \\
& \left.\cdot\left(\frac{\partial I_{D}}{\partial \alpha_{i 1}}\right) \rho_{\Delta V_{\mathrm{TH}}, \Delta \alpha_{i 1}} \sqrt{\sigma_{\Delta V_{\mathrm{TH}}}^{2}} \sqrt{\sigma_{\Delta \alpha_{i 1}}^{2}}\right]+2\left(\frac{\partial I_{D}}{\partial \beta}\right) \\
& \cdot\left(\frac{\partial I_{D}}{\partial \theta}\right) \rho_{\Delta \beta, \Delta \theta} \sqrt{\sigma_{\Delta \beta}^{2}} \sqrt{\sigma_{\Delta \theta}^{2}}+2\left(\frac{\partial I_{D}}{\partial \beta}\right)\left(\frac{\partial I_{D}}{\partial \alpha_{d 0}}\right) \\
& \cdot \rho_{\Delta \beta, \Delta \alpha_{d 0}} \sqrt{\sigma_{\Delta \beta}^{2}} \sqrt{\sigma_{\Delta \alpha_{d 0}}^{2}}+2\left(\frac{\partial I_{D}}{\partial \beta}\right)\left(\frac{\partial I_{D}}{\partial \alpha_{d 1}}\right) \\
& \cdot \rho_{\Delta \beta, \Delta \alpha_{d 1}} \sqrt{\sigma_{\Delta \beta}^{2}} \sqrt{\sigma_{\Delta \alpha_{d 1}}^{2}}+2\left(\frac{\partial I_{D}}{\partial \beta}\right)\left(\frac{\partial I_{D}}{\partial \alpha_{b 0}}\right) \\
& \cdot \rho_{\Delta \beta, \Delta \alpha_{b 0}} \sqrt{\sigma_{\Delta \beta}^{2}} \sqrt{\sigma_{\Delta \alpha_{b 0}}^{2}}+2\left(\frac{\partial I_{D}}{\partial \beta}\right)\left(\frac{\partial I_{D}}{\partial \alpha_{b 1}}\right) \\
& \cdot \rho_{\Delta \beta, \Delta \alpha_{b 1}} \sqrt{\sigma_{\Delta \beta}^{2}} \sqrt{\sigma_{\Delta \alpha_{b 1}}^{2}}+2 \sum_{i=1}^{N}\left[\left(\frac{\partial I_{D}}{\partial \beta}\right)\left(\frac{\partial I_{D}}{\partial \alpha_{i 0}}\right)\right. \\
& \left.\cdot \rho_{\Delta \beta, \Delta \alpha_{i 0} 0} \sqrt{\sigma_{\Delta \beta}^{2}} \sqrt{\sigma_{\Delta \alpha_{i 0}}^{2}}\right]+2 \sum_{i=1}^{N}\left[\left(\frac{\partial I_{D}}{\partial \beta}\right)\left(\frac{\partial I_{D}}{\partial \alpha_{i 1}}\right)\right. \\
& \left.\cdot \rho_{\Delta \beta, \Delta \alpha_{i 1}} \sqrt{\sigma_{\Delta \beta}^{2}} \sqrt{\sigma_{\Delta \alpha_{i 1}}^{2}}\right]+2\left(\frac{\partial I_{D}}{\partial \theta}\right)\left(\frac{\partial I_{D}}{\partial \alpha_{d 0}}\right) \\
& \cdot \rho_{\Delta \theta, \Delta \alpha_{d 0}} \sqrt{\sigma_{\Delta \theta}^{2}} \sqrt{\sigma_{\Delta \alpha_{d 0}}^{2}}+2\left(\frac{\partial I_{D}}{\partial \theta}\right)\left(\frac{\partial I_{D}}{\partial \alpha_{d 1}}\right) \\
& \cdot \rho_{\Delta \theta, \Delta \alpha_{d 1}} \sqrt{\sigma_{\Delta \theta}^{2}} \sqrt{\sigma_{\Delta \alpha_{d 1}}^{2}}+2\left(\frac{\partial I_{D}}{\partial \theta}\right)\left(\frac{\partial I_{D}}{\partial \alpha_{b 0}}\right) \\
& \cdot \rho_{\Delta \theta, \Delta \alpha_{b 0}} \sqrt{\sigma_{\Delta \theta}^{2}} \sqrt{\sigma_{\Delta \alpha_{b 0}}^{2}}+2\left(\frac{\partial I_{D}}{\partial \theta}\right)\left(\frac{\partial I_{D}}{\partial \alpha_{b 1}}\right) \\
& \cdot \rho_{\Delta \theta, \Delta \alpha_{b 1}} \sqrt{\sigma_{\Delta \theta}^{2}} \sqrt{\sigma_{\Delta \alpha_{b 1}}^{2}}+2 \sum_{i=1}^{N}\left[\left(\frac{\partial I_{D}}{\partial \theta}\right)\left(\frac{\partial I_{D}}{\partial \alpha_{i 0}}\right)\right. \\
& \left.\cdot \rho_{\Delta \alpha_{d 0}, \Delta \alpha_{i 1}} \sqrt{\sigma_{\Delta \alpha_{d 0}}^{2}} \sqrt{\sigma_{\Delta \alpha_{i 1}}^{2}}\right]+2\left(\frac{\partial I_{D}}{\partial \alpha_{d 1}}\right)\left(\frac{\partial I_{D}}{\partial \alpha_{b 0}}\right) \\
& \cdot \rho_{\Delta \alpha_{d 1}, \Delta \alpha_{b 0}} \sqrt{\sigma_{\Delta \alpha_{d 1}}^{2}} \sqrt{\sigma_{\Delta \alpha_{b 0}}^{2}}+2\left(\frac{\partial I_{D}}{\partial \alpha_{d 1}}\right)\left(\frac{\partial I_{D}}{\partial \alpha_{b 1}}\right) \\
& \cdot \rho_{\Delta \alpha_{d 1}, \Delta \alpha_{b 1}} \sqrt{\sigma_{\Delta \alpha_{d 1}}^{2}} \sqrt{\sigma_{\Delta \alpha_{b 1}}^{2}}+2 \sum_{i=1}^{N}\left[\left(\frac{\partial I_{D}}{\partial \alpha_{d 1}}\right)\left(\frac{\partial I_{D}}{\partial \alpha_{i 0}}\right)\right. \\
& \left.\cdot \rho_{\Delta \alpha_{d 1}, \Delta \alpha_{i 0}} \sqrt{\sigma_{\Delta \alpha_{d 1}}^{2}} \sqrt{\sigma_{\Delta \alpha_{i 0}}^{2}}\right]+2 \sum_{i=1}^{N}\left[\left(\frac{\partial I_{D}}{\partial \alpha_{d 1}}\right)\left(\frac{\partial I_{D}}{\partial \alpha_{i 1}}\right)\right. \\
& \left.\cdot \rho_{\Delta \alpha_{d 1}, \Delta \alpha_{i 1}} \sqrt{\sigma_{\Delta \alpha_{d 1}}^{2}} \sqrt{\sigma_{\Delta \alpha_{i 1}}^{2}}\right]+2\left(\frac{\partial I_{D}}{\partial \alpha_{b 0}}\right)\left(\frac{\partial I_{D}}{\partial \alpha_{b 1}}\right) \\
& \cdot \rho_{\Delta \alpha_{b 0}, \Delta \alpha_{b 1}} \sqrt{\sigma_{\Delta \alpha_{b 0}}^{2}} \sqrt{\sigma_{\Delta \alpha_{b 1}}^{2}}+2 \sum_{i=1}^{N}\left[\left(\frac{\partial I_{D}}{\partial \alpha_{b 0}}\right)\left(\frac{\partial I_{D}}{\partial \alpha_{i 0}}\right)\right. \\
& \left.\cdot \rho_{\Delta \alpha_{b 0}, \Delta \alpha_{i 0}} \sqrt{\sigma_{\Delta \alpha_{b 0}}^{2}} \sqrt{\sigma_{\Delta \alpha_{i 0}}^{2}}\right]+2 \sum_{i=1}^{N}\left[\left(\frac{\partial I_{D}}{\partial \alpha_{b 0}}\right)\left(\frac{\partial I_{D}}{\partial \alpha_{i 1}}\right)\right. \\
& \left.\cdot \rho_{\Delta \alpha_{b 0}, \Delta \alpha_{i 1}} \sqrt{\sigma_{\Delta \alpha_{b 0}}^{2}} \sqrt{\sigma_{\Delta \alpha_{i 1}}^{2}}\right]+2 \sum_{i=1}^{N}\left[\left(\frac{\partial I_{D}}{\partial \alpha_{b 1}}\right)\left(\frac{\partial I_{D}}{\partial \alpha_{i 0}}\right)\right. \\
& \left.\cdot \rho_{\Delta \alpha_{b 1}, \Delta \alpha_{i 0}} \sqrt{\sigma_{\Delta \alpha_{b 1}}^{2}} \sqrt{\sigma_{\Delta \alpha_{i 0}}^{2}}\right]+2 \sum_{i=1}^{N}\left[\left(\frac{\partial I_{D}}{\partial \alpha_{b 1}}\right)\left(\frac{\partial I_{D}}{\partial \alpha_{i 1}}\right)\right. \\
& \left.\cdot \rho_{\Delta \alpha_{b 1}, \Delta \alpha_{i 1}} \sqrt{\sigma_{\Delta \alpha_{b 1}}^{2}} \sqrt{\sigma_{\Delta \alpha_{i 1}}^{2}}\right]+2 \sum_{i=1}^{N}\left[\left(\frac{\partial I_{D}}{\partial \alpha_{i 0}}\right)\left(\frac{\partial I_{D}}{\partial \alpha_{i 1}}\right)\right. \\
& \left.\cdot \rho_{\Delta \alpha_{i 0}, \Delta \alpha_{i 1}} \sqrt{\sigma_{\Delta \alpha_{i 0}}^{2}} \sqrt{\sigma_{\Delta \alpha_{i 1}}^{2}}\right]+\sum_{\substack{j=1 \\
j \neq i}}^{N} \sum_{i=1}^{N}\left[\left(\frac{\partial I_{D}}{\partial \alpha_{i 0}}\right)\right. \\
& \left.\cdot\left(\frac{\partial I_{D}}{\partial \alpha_{j 0}}\right) \rho_{\Delta \alpha_{i 0}, \Delta \alpha_{j 0}} \sqrt{\sigma_{\Delta \alpha_{i 0}}^{2}} \sqrt{\sigma_{\Delta \alpha_{j 0}}^{2}}\right]
\end{aligned}
$$




$$
\begin{aligned}
& +\sum_{j=1}^{N} \sum_{i=1}^{N}\left[\left(\frac{\partial I_{D}}{\partial \alpha_{i 0}}\right)\left(\frac{\partial I_{D}}{\partial \alpha_{j 1}}\right) \rho_{\Delta \alpha_{i 0}, \Delta \alpha_{j 1}} \sqrt{\sigma_{\Delta \alpha_{i 0}}^{2}} \sqrt{\sigma_{\Delta \alpha_{j 1}}^{2}}\right] \\
& +\sum_{j=1}^{N} \sum_{i=1}^{N}\left[\left(\frac{\partial I_{D}}{\partial \alpha_{i 1}}\right)\left(\frac{\partial I_{D}}{\partial \alpha_{j 0}}\right) \rho_{\Delta \alpha_{i 1}, \Delta \alpha_{j 0}} \sqrt{\sigma_{\Delta \alpha_{i 1}}^{2}} \sqrt{\sigma_{\Delta \alpha_{j 0}}^{2}}\right] \\
& +\sum_{\substack{j=1 \\
j \neq i}}^{N} \sum_{i=1}^{N}\left[\left(\frac{\partial I_{D}}{\partial \alpha_{i 1}}\right)\left(\frac{\partial I_{D}}{\partial \alpha_{j 1}}\right)\right. \\
& \left.\cdot \rho_{\Delta \alpha_{i 1}, \Delta \alpha_{j 1}} \sqrt{\sigma_{\Delta \alpha_{i 1}}^{2}} \sqrt{\sigma_{\Delta \alpha_{j 1}}^{2}}\right],
\end{aligned}
$$

where $\rho_{\Delta x, \Delta y}$, for example, $\rho_{\Delta V_{\mathrm{TH}}, \Delta \beta}, \rho_{\Delta \beta, \Delta \theta}$, and $\rho_{\Delta V_{\mathrm{TH}}, \Delta \theta}$, denotes the correlation coefficient of $\Delta x$ and $\Delta y$ and displays their degree of statistical correlation. Moreover, $\sigma_{\Delta V_{\mathrm{TH}}}^{2}, \sigma_{\Delta \beta}^{2}$, $\sigma_{\Delta \alpha_{i 0}}^{2}, \sigma_{\Delta \alpha_{i 1}}^{2}, \sigma_{\Delta \alpha_{d 0}}^{2}, \sigma_{\Delta \alpha_{d 1}}^{2}, \sigma_{\Delta \alpha_{b 0}}^{2}, \sigma_{\Delta \alpha_{b 1}}^{2}$, and $\sigma_{\Delta \theta}^{2}$ are nonzero and, respectively, denote the variances of $\Delta V_{\mathrm{TH}}, \Delta \alpha_{i 0}, \Delta \alpha_{i 1}, \Delta \alpha_{d 0}$, $\Delta \alpha_{d 1}, \Delta \alpha_{b 0}, \Delta \alpha_{b 1}, \Delta \beta$, and $\Delta \theta$. Finally, it can be seen that the 1st up to 9 th terms of (25) have been contributed by $\sigma_{\Delta V_{\mathrm{TH}}}^{2}$, $\sigma_{\Delta \beta}^{2}, \sigma_{\Delta \alpha_{i 0}}^{2}, \sigma_{\Delta \alpha_{i 1}}^{2}, \sigma_{\Delta \alpha_{d 0}}^{2}, \sigma_{\Delta \alpha_{d 1}}^{2}, \sigma_{\Delta \alpha_{b 0}}^{2}, \sigma_{\Delta \alpha_{b 1}}^{2}$, and $\sigma_{\Delta \theta}^{2}$ where the others have been caused by the statistical correlations.

3.2. Saturation Region Part. Firstly, $I_{D}$ of FGMOSFET in this region will be formulated in a similar manner to that of FGMOSFET in triode where the second order effects have been taken into account as well. With the linear model of mobility degradation, $I_{D}$ of MOSFET in saturation can be given by

$$
\begin{aligned}
& I_{D} \\
& \quad=\frac{\beta}{2}\left[1-\theta\left(V_{\mathrm{GS}}-V_{\mathrm{TH}}\right)\right]\left(1+\lambda V_{\mathrm{DS}}\right)\left(V_{\mathrm{GS}}-V_{\mathrm{TH}}\right)^{2},
\end{aligned}
$$

where $\lambda$ stands for the channel length modulation coefficient which must be taken into account for MOSFET in saturation if the short channel effect has been considered.

For FGMOSFET in saturation region, the model formulation is simpler than that of triode FGMOSFET since $k_{i}, k_{\mathrm{fd}}$, $k_{\mathrm{fs}}$, and $k_{\mathrm{fb}}$ are constant. So, $V_{\mathrm{FG}}$ can be given by (3) and $I_{D}$ can be determined in a similar manner to that of FGMOSFET in triode by using (3) and (26) as given in (27) since $k_{\mathrm{fs}} \ll 1$ :

$$
\begin{aligned}
I_{D} & =\frac{\beta}{2}\left(1+\lambda V_{\mathrm{DS}}\right)[1 \\
& \left.-\theta\left(\sum_{i=1}^{N}\left[k_{i} V_{i}\right]+k_{\mathrm{fd}} V_{D}+k_{\mathrm{fb}} V_{B}-V_{S}-V_{\mathrm{TH}}\right)\right] \\
& \cdot\left(\sum_{i=1}^{N}\left[k_{i} V_{i}\right]\right. \\
& \left.+k_{\mathrm{fd}} V_{D}+k_{\mathrm{fb}} V_{B}-V_{S}-V_{\mathrm{TH}}\right)^{2} .
\end{aligned}
$$

As the process induced random variations in $\lambda(\Delta \lambda)$ must also be considered for FGMOSET in saturation region, $\Delta I_{D}$ is given by (28). By using (27), all derivatives can be found as (29)-(35):

$$
\begin{aligned}
& \Delta I_{D}=\left(\frac{\partial I_{D}}{\partial V_{\mathrm{TH}}}\right) \Delta V_{\mathrm{TH}}+\left(\frac{\partial I_{D}}{\partial \beta}\right) \Delta \beta+\left(\frac{\partial I_{D}}{\partial \theta}\right) \Delta \theta \\
& +\left(\frac{\partial I_{D}}{\partial \lambda}\right) \Delta \lambda+\left(\frac{\partial I_{D}}{\partial k_{\mathrm{fd}}}\right) \Delta k_{\mathrm{fd}}+\left(\frac{\partial I_{D}}{\partial k_{\mathrm{fb}}}\right) \Delta k_{\mathrm{fb}} \\
& +\sum_{i=1}^{N}\left[\left(\frac{\partial I_{D}}{\partial k_{i}}\right) \Delta k_{i}\right] \\
& \frac{\partial I_{D}}{\partial \beta}=\frac{1+\lambda V_{\mathrm{DS}}}{2}[1 \\
& \left.-\theta\left(\sum_{i=1}^{N}\left[k_{i} V_{i}\right]+k_{\mathrm{fd}} V_{D}+k_{\mathrm{fb}} V_{B}-V_{S}-V_{\mathrm{TH}}\right)\right] \\
& \cdot\left(\sum_{i=1}^{N}\left[k_{i} V_{i}\right]+k_{\mathrm{fd}} V_{D}+k_{\mathrm{fb}} V_{B}-V_{S}\right. \\
& \left.-V_{\mathrm{TH}}\right)^{2} \\
& \frac{\partial I_{D}}{\partial \theta}=\frac{\beta}{2}\left(1+\lambda V_{\mathrm{DS}}\right)\left(\sum_{i=1}^{N}\left[k_{i} V_{i}\right]+k_{\mathrm{fd}} V_{D}+k_{\mathrm{fb}} V_{B}-V_{S}\right. \\
& \left.-V_{\mathrm{TH}}\right)^{3} \\
& \frac{\partial I_{D}}{\partial V_{\mathrm{TH}}}=\frac{\beta}{2}\left(1+\lambda V_{\mathrm{DS}}\right) \\
& \cdot\left[3 \theta\left(\sum_{i=1}^{N}\left[k_{i} V_{i}\right]+k_{\mathrm{fd}} V_{D}+k_{\mathrm{fb}} V_{B}-V_{S}-V_{\mathrm{TH}}\right)\right. \\
& -2]\left(\sum_{i=1}^{N}\left[k_{i} V_{i}\right]+k_{\mathrm{fd}} V_{D}+k_{\mathrm{fb}} V_{B}-V_{S}-V_{\mathrm{TH}}\right), \\
& \frac{\partial I_{D}}{\partial \lambda}=\frac{\beta}{2} V_{\mathrm{DS}}[1 \\
& \left.-\theta\left(\sum_{i=1}^{N}\left[k_{i} V_{i}\right]+k_{\mathrm{fd}} V_{D}+k_{\mathrm{fb}} V_{B}-V_{S}-\mathrm{V}_{\mathrm{TH}}\right)\right] \\
& \cdot\left(\sum_{i=1}^{N}\left[k_{i} V_{i}\right]+k_{\mathrm{fd}} V_{D}+k_{\mathrm{fb}} V_{B}-V_{S}\right. \\
& \left.-V_{\mathrm{TH}}\right)^{2}
\end{aligned}
$$




$$
\begin{gathered}
\frac{\partial I_{D}}{\partial k_{\mathrm{fd}}}=-\frac{\beta}{2} V_{D}\left(1+\lambda V_{\mathrm{DS}}\right) \\
\cdot\left[3 \theta\left(\sum_{i=1}^{N}\left[k_{i} V_{i}\right]+k_{\mathrm{fd}} V_{D}+k_{\mathrm{fb}} V_{B}-V_{S}-V_{\mathrm{TH}}\right)\right. \\
-2]\left(\sum_{i=1}^{N}\left[k_{i} V_{i}\right]+k_{\mathrm{fd}} V_{D}+k_{\mathrm{fb}} V_{B}-V_{S}-V_{\mathrm{TH}}\right), \\
\frac{\partial I_{D}}{\partial k_{\mathrm{fb}}}=-\frac{\beta}{2} V_{B}\left(1+\lambda V_{\mathrm{DS}}\right) \\
\cdot\left[3 \theta\left(\sum_{i=1}^{N}\left[k_{i} V_{i}\right]+k_{\mathrm{fd}} V_{D}+k_{\mathrm{fb}} V_{B}-V_{S}-V_{\mathrm{TH}}\right)\right. \\
-2]\left(\sum_{i=1}^{N}\left[k_{i} V_{i}\right]+k_{\mathrm{fd}} V_{D}+k_{\mathrm{fb}} V_{B}-V_{S}-V_{\mathrm{TH}}\right), \\
\frac{\partial I_{D}}{\partial k_{i}}=-\frac{\beta}{2}\left(\sum_{i=1}^{N}\left[k_{i} V_{i}\right]\right)\left(1+\lambda V_{\mathrm{DS}}\right) \\
\cdot\left[3 \theta\left(\sum_{i=1}^{N}\left[k_{i} V_{i}\right]+k_{\mathrm{fd}} V_{D}+k_{\mathrm{fb}} V_{B}-V_{S}-V_{\mathrm{TH}}\right)\right. \\
-2]\left(\sum_{i=1}^{N}\left[k_{i} V_{i}\right]+k_{\mathrm{fd}} V_{D}+k_{\mathrm{fb}} V_{B}-V_{S}-V_{\mathrm{TH}}\right) .
\end{gathered}
$$

Similarly to $\Delta I_{D}$ of the FGMOSFET in triode, $\Delta I_{D}$ of the FGMOSFET in saturation is also a random variable. So, its statistical behavior must be analytically modeled as well. Since $\Delta V_{\mathrm{TH}}, \Delta \beta, \Delta k_{i}, \Delta k_{\mathrm{fd}}, \Delta k_{\mathrm{fb}}, \Delta \theta$, and $\Delta \lambda$ have zero means, it has also been found that $\Delta I_{D, \text { avr }}=0$. Moreover, $\sigma_{\Delta I_{D}}^{2}$ can be given by taking the statistical correlations of $\Delta V_{\mathrm{TH}}, \Delta \beta, \Delta k_{i}$, $\Delta k_{\mathrm{fd}}, \Delta k_{\mathrm{fb}}, \Delta \theta$, and $\Delta \lambda$ into account as given in (36) where the first seven terms have been, respectively, contributed by $\sigma_{\Delta V_{\mathrm{TH}}}^{2}, \sigma_{\Delta \beta}^{2}, \sigma_{\Delta k_{i}}^{2}, \sigma_{\Delta k_{\mathrm{fd}}}^{2}, \sigma_{\Delta k_{\mathrm{fb}}}^{2}, \sigma_{\Delta \theta}^{2}$, and $\sigma_{\Delta \lambda}^{2}$. On the other hand, the other terms have been contributed by the statistical correlations.

Before proceeding to the model verification, it should be mentioned here that $\rho_{\Delta x, \Delta y}$ can be obtained either by using the scatter plot $[25,26]$ or by calculation as given in (37) which can be obtained based on the prior knowledge that the averages of $\Delta x$ and $\Delta y$ are 0 as they are process induced device level random variations, that is, $\Delta V_{\mathrm{TH}}, \Delta \beta$, and $\Delta \theta$. Moreover, $E[$ ] stands for the expectation operator:

$$
\begin{aligned}
& \sigma_{\Delta I_{D}}^{2} \\
& =\left(\frac{\partial I_{D}}{\partial V_{\mathrm{TH}}}\right)^{2} \sigma_{\Delta V_{\mathrm{TH}}}^{2}+\left(\frac{\partial I_{D}}{\partial \beta}\right)^{2} \sigma_{\Delta \beta}^{2}+\left(\frac{\partial I_{D}}{\partial \theta}\right)^{2} \sigma_{\Delta \theta}^{2} \\
& \quad+\left(\frac{\partial I_{D}}{\partial \lambda}\right)^{2} \sigma_{\Delta \lambda}^{2}+\left(\frac{\partial I_{D}}{\partial k_{\mathrm{fb}}}\right)^{2} \sigma_{\Delta k_{\mathrm{fb}}}^{2}+\left(\frac{\partial I_{D}}{\partial k_{\mathrm{fd}}}\right)^{2} \sigma_{\Delta k_{\mathrm{fd}}}^{2} \\
& \quad+\sum_{i=1}^{N}\left[\left(\frac{\partial I_{D}}{\partial k_{i}}\right)^{2} \sigma_{\Delta k_{i}}^{2}\right]
\end{aligned}
$$

$$
\begin{aligned}
& +2\left(\frac{\partial I_{D}}{\partial \beta}\right)\left(\frac{\partial I_{D}}{\partial V_{\mathrm{TH}}}\right) \rho_{\Delta \beta, \Delta V_{\mathrm{TH}}} \sqrt{\sigma_{\Delta \beta}^{2}} \sqrt{\sigma_{\Delta V_{\mathrm{TH}}}^{2}} \\
& +2\left(\frac{\partial I_{D}}{\partial \beta}\right)\left(\frac{\partial I_{D}}{\partial \theta}\right) \rho_{\Delta \beta, \Delta \theta} \sqrt{\sigma_{\Delta \beta}^{2}} \sqrt{\sigma_{\Delta \theta}^{2}} \\
& +2\left(\frac{\partial I_{D}}{\partial \beta}\right)\left(\frac{\partial I_{D}}{\partial \lambda}\right) \rho_{\Delta \beta, \Delta \lambda} \sqrt{\sigma_{\Delta \beta}^{2}} \sqrt{\sigma_{\Delta \lambda}^{2}} \\
& +2\left(\frac{\partial I_{D}}{\partial \beta}\right)\left(\frac{\partial I_{D}}{\partial k_{\mathrm{fd}}}\right) \rho_{\Delta \beta, \Delta k_{\mathrm{fd}}} \sqrt{\sigma_{\Delta \beta}^{2}} \sqrt{\sigma_{\Delta k_{\mathrm{fd}}}^{2}} \\
& +2\left(\frac{\partial I_{D}}{\partial \beta}\right)\left(\frac{\partial I_{D}}{\partial k_{\mathrm{fb}}}\right) \rho_{\Delta \beta, \Delta k_{\mathrm{fb}}} \sqrt{\sigma_{\Delta \beta}^{2}} \sqrt{\sigma_{\Delta k_{\mathrm{fb}}}^{2}} \\
& +2 \sum_{i=1}^{N}\left[\left(\frac{\partial I_{D}}{\partial \beta}\right)\left(\frac{\partial I_{D}}{\partial k_{i}}\right) \rho_{\Delta \beta, \Delta k_{i}} \sqrt{\sigma_{\Delta \beta}^{2}} \sqrt{\sigma_{\Delta k_{i}}^{2}}\right] \\
& +2\left(\frac{\partial I_{D}}{\partial \theta}\right)\left(\frac{\partial I_{D}}{\partial V_{\mathrm{TH}}}\right) \rho_{\Delta \theta, \Delta V_{\mathrm{TH}}} \sqrt{\sigma_{\Delta \theta}^{2}} \sqrt{\sigma_{\Delta V_{\mathrm{TH}}}^{2}} \\
& +2\left(\frac{\partial I_{D}}{\partial \theta}\right)\left(\frac{\partial I_{D}}{\partial \lambda}\right) \rho_{\Delta \theta, \Delta \lambda} \sqrt{\sigma_{\Delta \theta}^{2}} \sqrt{\sigma_{\Delta \lambda}^{2}} \\
& +2\left(\frac{\partial I_{D}}{\partial \theta}\right)\left(\frac{\partial I_{D}}{\partial k_{\mathrm{fd}}}\right) \rho_{\Delta \theta, \Delta k_{\mathrm{fd}}} \sqrt{\sigma_{\Delta \theta}^{2}} \sqrt{\sigma_{\Delta k_{\mathrm{fd}}}^{2}} \\
& +2\left(\frac{\partial I_{D}}{\partial \theta}\right)\left(\frac{\partial I_{D}}{\partial k_{\mathrm{fb}}}\right) \rho_{\Delta \theta, \Delta k_{\mathrm{fb}}} \sqrt{\sigma_{\Delta \theta}^{2}} \sqrt{\sigma_{\Delta k_{\mathrm{fb}}}^{2}} \\
& +2 \sum_{i=1}^{N}\left[\left(\frac{\partial I_{D}}{\partial \theta}\right)\left(\frac{\partial I_{D}}{\partial k_{i}}\right) \rho_{\Delta \theta, \Delta k_{i}} \sqrt{\sigma_{\Delta \theta}^{2}} \sqrt{\sigma_{\Delta k_{i}}^{2}}\right] \\
& +2\left(\frac{\partial I_{D}}{\partial V_{\mathrm{TH}}}\right)\left(\frac{\partial I_{D}}{\partial \lambda}\right) \rho_{\Delta V_{\mathrm{TH}}, \Delta \alpha_{\lambda}} \sqrt{\sigma_{\Delta V_{\mathrm{TH}}}^{2}} \sqrt{\sigma_{\Delta \lambda}^{2}} \\
& +2\left(\frac{\partial I_{D}}{\partial V_{\mathrm{TH}}}\right)\left(\frac{\partial I_{D}}{\partial k_{\mathrm{fd}}}\right) \rho_{\Delta V_{\mathrm{TH}}, \Delta k_{\mathrm{fd}}} \sqrt{\sigma_{\Delta V_{\mathrm{TH}}}^{2}} \sqrt{\sigma_{\Delta k_{\mathrm{fd}}}^{2}} \\
& +2\left(\frac{\partial I_{D}}{\partial V_{\mathrm{TH}}}\right)\left(\frac{\partial I_{D}}{\partial k_{\mathrm{fb}}}\right) \rho_{\Delta V_{\mathrm{TH}}, \Delta k_{\mathrm{fb}}} \sqrt{\sigma_{\Delta V_{\mathrm{TH}}}^{2}} \sqrt{\sigma_{\Delta \alpha k_{\mathrm{fb}}}^{2}} \\
& +2 \sum_{i=1}^{N}\left[\left(\frac{\partial I_{D}}{\partial V_{\mathrm{TH}}}\right)\left(\frac{\partial I_{D}}{\partial k_{i}}\right) \rho_{\Delta V_{\mathrm{TH}}, \Delta k_{i}} \sqrt{\sigma_{\Delta V_{\mathrm{TH}}}^{2}} \sqrt{\sigma_{\Delta k_{i}}^{2}}\right] \\
& +2\left(\frac{\partial I_{D}}{\partial \lambda}\right)\left(\frac{\partial I_{D}}{\partial k_{\mathrm{fd}}}\right) \rho_{\Delta \lambda, \Delta k_{\mathrm{fd}}} \sqrt{\sigma_{\Delta \lambda}^{2}} \sqrt{\sigma_{\Delta \alpha k_{\mathrm{fd}}}^{2}} \\
& +2\left(\frac{\partial I_{D}}{\partial \lambda}\right)\left(\frac{\partial I_{D}}{\partial k_{\mathrm{fb}}}\right) \rho_{\Delta \lambda, \Delta k_{\mathrm{fb}}} \sqrt{\sigma_{\Delta \lambda}^{2}} \sqrt{\sigma_{\Delta \alpha k_{\mathrm{fb}}}^{2}} \\
& +2 \sum_{i=1}^{N}\left[\left(\frac{\partial I_{D}}{\partial \lambda}\right)\left(\frac{\partial I_{D}}{\partial k_{i}}\right) \rho_{\Delta \lambda, \Delta k_{i}} \sqrt{\sigma_{\Delta \lambda}^{2}} \sqrt{\sigma_{\Delta k_{i}}^{2}}\right] \\
& +2\left(\frac{\partial I_{D}}{\partial k_{\mathrm{fd}}}\right)\left(\frac{\partial I_{D}}{\partial k_{\mathrm{fb}}}\right) \rho_{\Delta k_{\mathrm{fd}}, \Delta k_{\mathrm{fb}}} \sqrt{\sigma_{\Delta k_{\mathrm{fd}}}^{2}} \sqrt{\sigma_{\Delta \alpha k_{\mathrm{fb}}}^{2}} \\
& +2 \sum_{i=1}^{N}\left[\left(\frac{\partial I_{D}}{\partial k_{\mathrm{fd}}}\right)\left(\frac{\partial I_{D}}{\partial k_{i}}\right) \rho_{\Delta k_{\mathrm{fd}}, \Delta k_{i}} \sqrt{\sigma_{\Delta k_{\mathrm{fd}}}^{2}} \sqrt{\sigma_{\Delta k_{i}}^{2}}\right]
\end{aligned}
$$




$$
\begin{gathered}
+2 \sum_{i=1}^{N}\left[\left(\frac{\partial I_{D}}{\partial k_{\mathrm{fb}}}\right)\left(\frac{\partial I_{D}}{\partial k_{i}}\right) \rho_{\Delta k_{\mathrm{fb}}, \Delta k_{i}} \sqrt{\sigma_{\Delta k_{\mathrm{fb}}}^{2}} \sqrt{\sigma_{\Delta k_{i}}^{2}}\right] \\
+\sum_{\substack{j=1 \\
j \neq i}}^{N} \sum_{i=1}^{N}\left[\left(\frac{\partial I_{D}}{\partial k_{i}}\right)\left(\frac{\partial I_{D}}{\partial k_{j}}\right) \rho_{\Delta k_{i}, \Delta k_{j}} \sqrt{\sigma_{\Delta k_{i}}^{2}} \sqrt{\sigma_{\Delta k_{j}}^{2}}\right] \\
\rho_{\Delta x, \Delta y}=\frac{E[\Delta x \Delta y]}{\sigma_{\Delta x} \sigma_{\Delta y}}
\end{gathered}
$$

\section{Model Verification}

Before proceeding further, it should be mentioned here that the model verification has been performed by assuming that $C_{i} \gg C_{\mathrm{fd}}, C_{\mathrm{fs}}, C_{\mathrm{fb}}$ as has been assumed in many previous studies on FGMOSFET, for example, $[2,7-9,20]$. As a result, $k_{\mathrm{fd}}, k_{\mathrm{fs}}$, and $k_{\mathrm{fb}}$ can be neglected as $k_{i} \gg k_{\mathrm{fd}}, k_{\mathrm{fs}}, k_{\mathrm{fb}}$ and $C_{T}$ is now independent of $V_{D}$ because $C_{\mathrm{fb}}$ which causes the dependency of $C_{T}$ on $V_{D}$ can be neglected. So, $k_{i}$, which depends on $C_{T}$, is now constant for both triode and saturation regions as also assumed in [22].

Moreover, the model verification has been performed based on FGMOSFET of both N-type and P-type with $N=2$ and $k_{1}=k_{2}=0.5$ for both triode and saturation regions, $W / L$ $=20 / 0.25$, SPICE BSIM3v3, and $0.25 \mu \mathrm{m}$ level CMOS process technology of TSMC where all necessary parameters have been provided by MOSIS. For performing the verification, the root mean square (rms.) value of $\Delta I_{D}$ calculated by using the model $\left(\Delta I_{D, \mathrm{rms}, M}\right)$ has been graphically compared to its SPICE BSIM3v3 based reference $\left(\Delta I_{D, \text { rms,SPICE }}\right)$ obtained by using the Monte-Carlo SPICE simulation with 1000 runs. For convenience, $\Delta V_{\mathrm{TH}}, \Delta \beta, \Delta k_{1}, \Delta k_{2}, \Delta \theta$, and $\Delta \lambda$ have been assumed to be normally distributed and all $\rho_{\Delta x, \Delta y}$ 's have been assumed to be 0.5 which is a reasonable estimation [26]. Since correlations must be taken into account in the simulations, each of $\Delta V_{\mathrm{TH}}, \Delta \beta, \Delta k_{1}, \Delta k_{2}, \Delta \theta$, and $\Delta \lambda$ has been expressed as a weighted sum of its correlated and uncorrelated components which are both normally distributed [26] and which have equal weights given by $\sqrt{0.5}$ due to the assumed $\rho_{\Delta x, \Delta y}$ 's.

Finally, the SPICE BSIM3v3 based modelling of FGMOSFET with $N=2$ can be performed by using the two inputs' version of the equivalent circuit of FGMOSFET in Figure 2 where the core MOSFET has been modelled by using the SPICE BSIM3v3 and the simulation methodology proposed in [23] has been adopted for solving the convergence problem of the simulator. Both $\Delta I_{D, \mathrm{rms}, M}$ and $\Delta I_{D, \text { rms,SPICE }}$ have been expressed as percentage of deterministic $I_{D}$ and comparatively plotted against the magnitude of the voltage of the 1st and 2nd inputs of FGMOSFET which are ranged from 3 to $8 \mathrm{~V}$ and denoted by $\left|V_{1}\right|$ and $\left|V_{2}\right|$, respectively. It should be mentioned here that $\left|V_{2}\right|=0 \mathrm{~V}$ in the comparative plots against $\left|V_{1}\right|$ and vice versa. At this point, verification of the proposed model will be presented.

4.1. Verification of Triode Region Part. Since $\lambda$ and of course $\Delta \lambda$ have not been considered in triode region, only $\Delta V_{\mathrm{TH}}$,

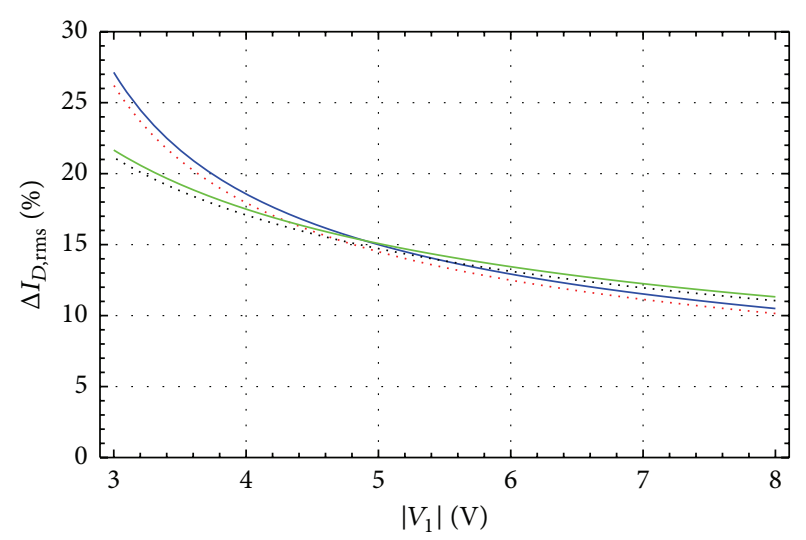

FIGURE 3: Triode and saturation regions N-type FGMOSFET based comparative plots of $\Delta I_{D, \mathrm{rms}, M}$ and $\Delta I_{D, \text { rms,SPICE }}$ against $\left|V_{1}\right|$ where $\left|V_{2}\right|=0$.

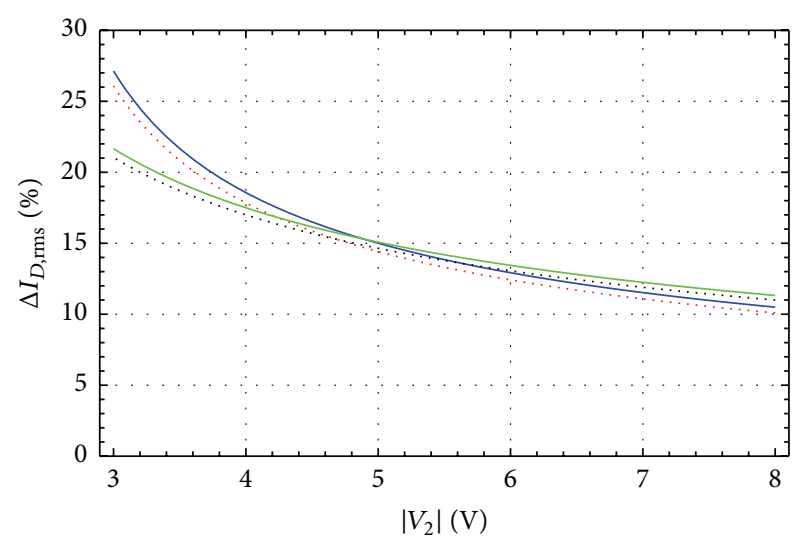

FIGURE 4: Triode and saturation regions N-type FGMOSFET based comparative plots of $\Delta I_{D, \mathrm{rms}, M}$ and $\Delta I_{D, \mathrm{rms}, \mathrm{SPICE}}$ against $\left|V_{2}\right|$ where $\left|V_{1}\right|=0$.

$\Delta \beta, \Delta \theta, \Delta k_{1}$, and $\Delta k_{2}$ have been taken into account. We let $\sigma_{\Delta V_{\mathrm{TH}}} / V_{\mathrm{TH}}=\sigma_{\Delta \beta} / \beta=\sigma_{\Delta \theta} / \theta=\sigma_{\Delta k_{1}} / k_{1}=\sigma_{\Delta k_{2}} / k_{2}$ for making the device level variations be bias-free and let these quantities be 0.01 as it has been assumed that $V_{\mathrm{TH}}, \beta, \theta, k_{1}$, and $k_{2}$ have $1 \%$ variation. For N-type FGMOSFET, the comparative plots of $\Delta I_{D, \text { rms, } M}$ and $\Delta I_{D, \text { rms,SPICE }}$ against $\left|V_{1}\right|$ where $\left|V_{2}\right|=$ 0 and vice versa can be, respectively, shown in Figures 3 and 4 whereas those of P-type FGMOSFET are shown in Figures 5 and 6 . In these figures, highly strong agreements between $\Delta I_{D, \mathrm{rms}, M}$ and $\Delta I_{D, \mathrm{rms}, \text { SPICE }}$ which are, respectively, drawn as blue normal curves and red dotted curves can be observed and the average deviations of $\Delta I_{D, \mathrm{rms}, M}$ from $\Delta I_{D, \mathrm{rms} \text {,SPICE }}$ have been found as $3.74725 \%$ and $3.70765 \%$ for N-type and P-type FGMOSFET, respectively. These deviations which can be determined from triode region $\mathrm{N}$-type and P-type FGMOSFET based comparative plots shown in Figures 3 and 4 and in Figures 5 and 6, respectively, are notably very small. So, it can be seen that triode region part of the proposed model has been found to be very accurate.

4.2. Verification of Saturation Region Part. In saturation region, $\lambda$ and $\Delta \lambda$ must be considered. For making the device 


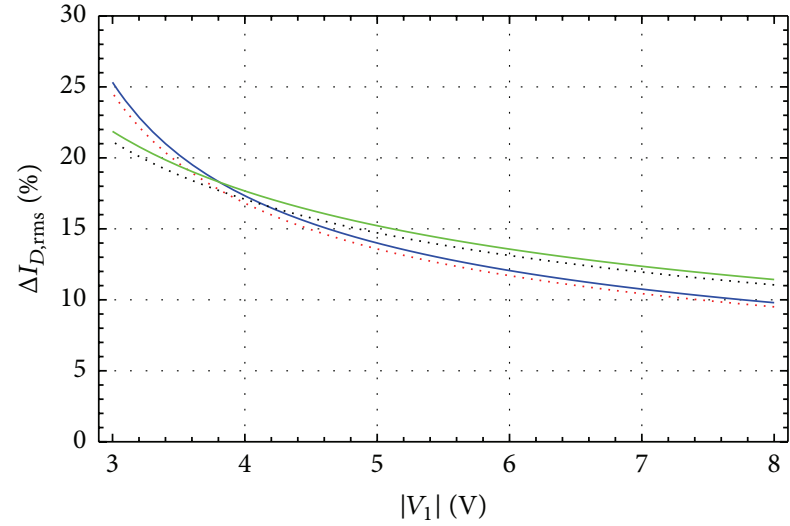

FIGURE 5: Triode and saturation regions P-type FGMOSFET based comparative plots of $\Delta I_{D, \mathrm{rms}, M}$ and $\Delta I_{D, \mathrm{rms}, \text { SPICE }}$ against $\left|V_{1}\right|$ where $\left|V_{2}\right|=0$.

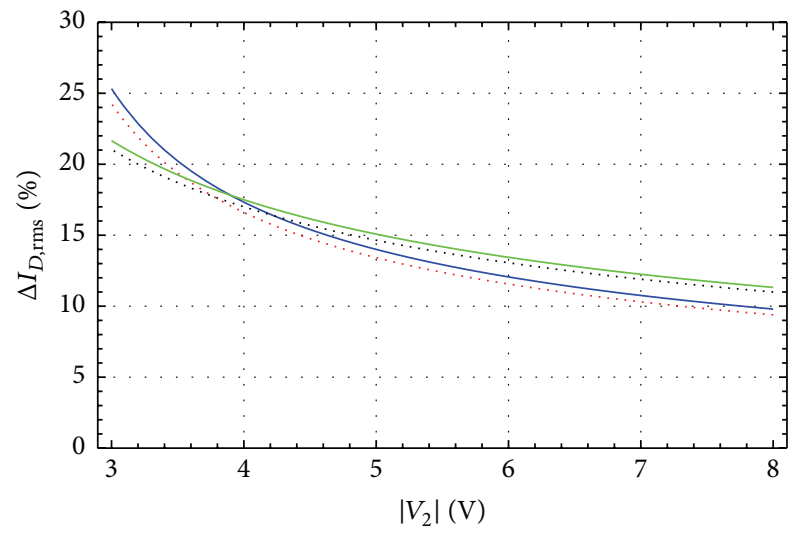

FIgURE 6: Triode and saturation regions P-type FGMOSFET based comparative plots of $\Delta I_{D, \mathrm{rms}, M}$ and $\Delta I_{D, \mathrm{rms} \text { SPICE }}$ against $\left|V_{2}\right|$ where $\left|V_{1}\right|=0$.

level variations be bias-free and following the assumption that $V_{\mathrm{TH}}, \beta, \theta, k_{1}, k_{2}$, and $\lambda$ have $1 \%$ variation, we let $\sigma_{\Delta V_{\mathrm{TH}}} / V_{\mathrm{TH}}=$ $\sigma_{\Delta \beta} / \beta=\sigma_{\Delta \lambda} / \lambda=\sigma_{\Delta \theta} / \theta=\sigma_{\Delta k_{1}} / k_{1}=\sigma_{\Delta k_{2}} / k_{2}=0.01$. The N-type FGMOSFET based comparative plots of $\Delta I_{D, \mathrm{rms}, M}$ and $\Delta I_{D \text {,rms,SPICE }}$ against $\left|V_{1}\right|$ where $\left|V_{2}\right|=0$ and vice versa can also be, respectively, depicted in Figures 3 and 4 where those based on P-type device are shown in Figures 5 and 6 as well. However, $\Delta I_{D, \mathrm{rms}, M}$ and $\Delta I_{D, \mathrm{rms}, \text { SPICE }}$ have been, respectively, drawn as green normal curves and black dotted curves instead in this case. From Figures 3-6, highly strong agreements between $\Delta I_{D, \mathrm{rms}, M}$ and $\Delta I_{D, \mathrm{rms}, \mathrm{SPICE}}$ can also be seen in this case. The average deviations between $\Delta I_{D, \mathrm{rms}, M}$ and $\Delta I_{D, \text { rms,SPICE }}$ have been, respectively, found as $2.6901 \%$ and $3.6681 \%$ for N-type and P-type FGMOSFET. These deviations which can be determined from the saturation region FGMOSFET based comparative plots are also considerably very small. As a result, the accuracy of saturation region part of the model has been verified.

From Figures 3-6, it has been found that both $\Delta I_{D, \mathrm{rms}, M}$ and $\Delta I_{D, \text { rms,SPICE }}$ are increased with decreasing $\left|V_{1}\right|$ and $\left|V_{2}\right|$ because they have been expressed as percentage of their corresponding deterministic $I_{D}$ 's which are increased with increasing $\left|V_{1}\right|$ and $\left|V_{2}\right|$ but with larger degree than the gross values of both rms. values do. Such increasing of $\Delta I_{D, \mathrm{rms}, M}$ and $\Delta I_{D, \text { rms,SPICE }}$ with decreasing $\left|V_{1}\right|$ and $\left|V_{2}\right|$ means that $\Delta I_{D}$ become more critical in low voltage/low power condition.

By considering the magnitude of both $\Delta I_{D, \mathrm{rms}, M}$ and $\Delta I_{D, \text { rms,SPICE }}$, it has also been found that the FGMOSFET in triode is more robust than that in saturation region when $\left|V_{1}\right|$ and $\left|V_{2}\right|$ become sufficiently large. This can be obviously seen in P-type device. On the other hand, the device in saturation is more robust when $\left|V_{1}\right|$ and $\left|V_{2}\right|$ become adequately low. This is more obvious in the N-type FGMOSFET. If desired, $\Delta I_{D, \mathrm{rms}, M}$ can fit $\Delta I_{D, \mathrm{rms}, \mathrm{SPICE}}$ simulated by using BSIM4. In order to do so, the optimum parameters of MOSFET's $I_{D}$ equations, that is, (6) and (14), extracted from BSIM4's $I_{D}$ model by using the optimization algorithm [24] must be used.

\section{Discussions}

Firstly, insight into device level variability of FGMOSFET will be discussed. By using the proposed model, the rate of change of per unit $\Delta I_{D}\left(\Delta I_{D} / I_{D}\right)$ of FGMOSFET in triode region with respect to per unit $\Delta V_{\mathrm{TH}}, \Delta \alpha_{i 0}, \Delta \alpha_{i 1}, \Delta \alpha_{d 0}, \Delta \alpha_{d 1}$, $\Delta \alpha_{b 0}, \Delta \alpha_{b 1}, \Delta \beta$, and $\Delta \theta$ denoted by $\Delta V_{\mathrm{TH}} / V_{\mathrm{TH}}, \Delta \alpha_{i 0} / \alpha_{i 0}$, $\Delta \alpha_{i 1} / \alpha_{i 1}, \Delta \alpha_{d 0} / \alpha_{d 0}, \Delta \alpha_{d 1} / \alpha_{d 1}, \Delta \alpha_{b 0} / \alpha_{b 0}, \Delta \alpha_{b 1} / \alpha_{b 1}, \Delta \beta / \beta$, and $\Delta \theta / \theta$, respectively, can be approximately given under low voltage/low power condition as follows:

$$
\begin{aligned}
\frac{\partial \Delta I_{D} / I_{D}}{\partial \Delta V_{\mathrm{TH}} / V_{\mathrm{TH}}} & =\frac{1+2 \theta V_{\mathrm{TH}}}{1+\theta V_{\mathrm{TH}}}, \\
\frac{\partial \Delta I_{D} / I_{D}}{\partial \Delta \beta / \beta} & =1, \\
\frac{\partial \Delta I_{D} / I_{D}}{\partial \Delta \theta / \theta} & =\frac{\theta V_{\mathrm{TH}}}{1+\theta V_{\mathrm{TH}}}, \\
\frac{\partial \Delta I_{D} / I_{D}}{\partial \Delta \alpha_{d 0} / \alpha_{d 0}} & =0 \\
\frac{\partial \Delta I_{D} / I_{D}}{\partial \Delta \alpha_{d 1} / \alpha_{d 1}} & =0 \\
\frac{\partial \Delta I_{D} / I_{D}}{\partial \Delta \alpha_{b 0} / \alpha_{b 0}} & =0 \\
\frac{\partial \Delta I_{D} / I_{D}}{\partial \Delta \alpha_{b 1} / \alpha_{b 1}} & =0 \\
\frac{\partial \Delta I_{D} / I_{D}}{\partial \Delta \alpha_{i 0} / \alpha_{i 0}} & =0 \\
\frac{\partial \Delta I_{D} / I_{D}}{\partial \Delta \alpha_{i 1} / \alpha_{i 1}} & =0 .
\end{aligned}
$$

For the saturation region, a similar rate of changes can be approximately found under a similar condition as

$$
\frac{\partial \Delta I_{D} / I_{D}}{\partial \Delta \beta / \beta}=1
$$




$$
\begin{aligned}
\frac{\partial \Delta I_{D} / I_{D}}{\partial \Delta \theta / \theta} & =\frac{\theta V_{\mathrm{TH}}}{1+\theta V_{\mathrm{TH}}}, \\
\frac{\partial \Delta I_{D} / I_{D}}{\partial \Delta V_{\mathrm{TH}} / V_{\mathrm{TH}}} & =\frac{2+3 \theta V_{\mathrm{TH}}}{1+\theta V_{\mathrm{TH}}}, \\
\frac{\partial \Delta I_{D} / I_{D}}{\partial \Delta \lambda / \lambda} & =0, \\
\frac{\partial \Delta I_{D} / I_{D}}{\partial \Delta k_{\mathrm{fd}} / k_{\mathrm{fd}}} & =0, \\
\frac{\partial \Delta I_{D} / I_{D}}{\partial \Delta k_{\mathrm{fb}} / k_{\mathrm{fb}}} & =0, \\
\frac{\partial \Delta I_{D} / I_{D}}{\partial \Delta k_{i} / k_{i}} & =0 .
\end{aligned}
$$

From these equations, it can be seen that all process induced device level random variations are relatively insignificant compared to $\Delta \beta, \Delta \theta$, and $\Delta V_{\mathrm{TH}}$ under low voltage/low power condition. So, $\Delta \beta, \Delta \theta$, and $\Delta V_{\mathrm{TH}}$ must be mostly considered in the variability aware design of low voltage/low power FGMOSFET based circuit. Moreover, the effects of $\Delta \theta$ and $\Delta V_{\mathrm{TH}}$ can be reduced by lowering either $\theta$ or $V_{\mathrm{TH}}$ or both as can be seen from (38), (40), (48), and (49).

Secondly, application of the proposed model will be discussed. This model can be used as the basis for the analytical modeling of process induced random variation in the circuit level parameter of any FGMOSFET based circuit. Let the circuit level parameter of interest of any FGMOSFET based circuit which is composed of $M$ FGMOSFETs be $X$. The process induced random variation in $X(\Delta X)$ caused by the combined effect of $\Delta I_{D}$ of each FGMOSFET can be given by

$$
\Delta X=\sum_{m=1}^{M}\left[\frac{\partial X}{\partial I_{D m}} \Delta I_{D m}\right]
$$

where $I_{D m}$ and $\Delta I_{D m}$ denote the $I_{D}$ and $\Delta I_{D}$ of any $m$ th FGMOSFET. Since $\Delta I_{D m}$ can be analytically determined by using the proposed model due to its definition, so does $\Delta X$ via (54).

Since the average of $\Delta X$ is zero as those of $\Delta I_{D m}$ 's are, it is convenient to analytically model the statistical behavior of $\Delta X$ by using its variance $\left(\sigma_{\Delta X}^{2}\right)$ as those of $\Delta I_{D m}$ 's are nonzero. According to the above definition of $\Delta I_{D m}$, the variance of $\Delta I_{D m}\left(\sigma_{\Delta I_{D m}}^{2}\right)$ can be determined by using the proposed model. So, $\sigma_{\Delta X}^{2}$ can be determined by using the proposed model as well as with the aid of the following equation:

$$
\begin{gathered}
\sigma_{\Delta X}^{2}=\sum_{m=1}^{M}\left[\left(\frac{\partial X}{\partial I_{D m}}\right)^{2} \sigma_{\Delta I_{D m}}^{2}\right]+\sum_{\substack{m=1 \\
m \neq k}}^{M} \sum_{\substack{m=1\\
}}^{M}\left[\left(\frac{\partial X}{\partial I_{D m}}\right)\right. \\
\left.\cdot\left(\frac{\partial X}{\partial I_{D k}}\right) \rho_{I_{D m}, I_{D k}} \sqrt{\sigma_{\Delta I_{D m}}^{2} \sigma_{\Delta I_{D k}}^{2}}\right] .
\end{gathered}
$$

As a practical illustrative example, let $X$ be the transconductance $\left(G_{m}\right)$ of the FGMOSFET based voltage to current converter (VIC) [1]. The equivalent circuit of its core circuit

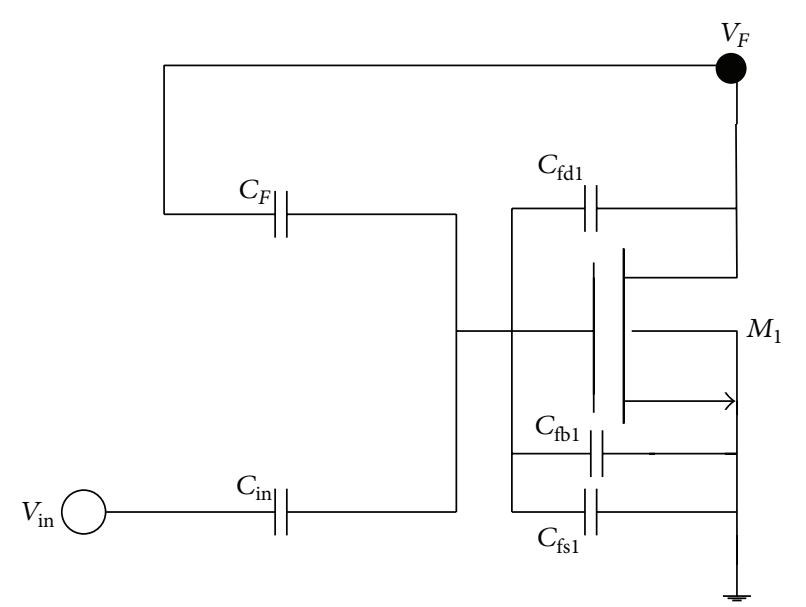

FIGURE 7: Equivalent circuit of FGMOSFET based transconductor which is the core circuit of FGMOSFET based VIC [1].

which is the FGMOSFET based transconductor can be depicted as in Figure 7 where $C_{F}$ and $C_{\text {in }}$ are the feedback and input capacitance of the transconductor [1]. Moreover, $C_{\mathrm{fd} 1}$, $C_{\mathrm{fs} 1}$, and $C_{\mathrm{fb} 1}$ are, respectively, $C_{\mathrm{fd}}, C_{\mathrm{fs}}$, and $C_{\mathrm{fb}}$ of $M_{1}$.

Obviously, $G_{m}$ can be given by

$$
G_{m}=\frac{\sqrt{2 \beta_{1}} C_{\mathrm{in}}}{C_{\mathrm{in}}+C_{F}+C_{\mathrm{fd} 1}+C_{\mathrm{fs} 1}+C_{\mathrm{fb} 1}} \sqrt{I_{D 1}},
$$

where $I_{D 1}$ and $\beta_{1}$ denote $I_{D}$ and $\beta$ of $M_{1}$.

Since $M_{1}$ is in saturation region and has $N=2, I_{D 1}$ can be given as follows:

$$
\begin{aligned}
I_{D 1}= & \frac{\beta_{1}}{2}\left(1+\lambda_{1} V_{f}\right) \\
& \cdot\left[1-\theta_{1}\left(k_{\mathrm{in}} V_{\mathrm{in}}+\left(k_{F}+k_{\mathrm{fd} 1}\right) V_{F}-\mathrm{V}_{\mathrm{TH} 1}\right)\right] \\
& \cdot\left(k_{\mathrm{in}} V_{\mathrm{in}}+\left(k_{F}+k_{\mathrm{fd} 1}\right) V_{F}-\mathrm{V}_{\mathrm{TH} 1}\right)^{2},
\end{aligned}
$$

where $V_{\mathrm{TH} 1}, \theta_{1}$, and $\lambda_{1}$ denote $V_{\mathrm{TH}}, \theta$, and $\lambda$ of $M_{1}$.

Moreover, $k_{\mathrm{in}}, k_{F}$, and $k_{\mathrm{fd} 1}$ can be defined as

$$
\begin{aligned}
k_{\mathrm{in}} & =\frac{C_{\mathrm{in}}}{C_{\mathrm{in}}+C_{F}+C_{\mathrm{fd} 1}+C_{\mathrm{fs} 1}+C_{\mathrm{fb} 1}}, \\
k_{F} & =\frac{C_{F}}{C_{\mathrm{in}}+C_{F}+C_{\mathrm{fd} 1}+C_{\mathrm{fs} 1}+C_{\mathrm{fb} 1}}, \\
k_{\mathrm{fd} 1} & =\frac{C_{\mathrm{fd} 1}}{C_{\mathrm{in}}+C_{F}+C_{\mathrm{fd} 1}+C_{\mathrm{fs} 1}+C_{\mathrm{fb} 1}} .
\end{aligned}
$$

At this point, the analytical model of process induced random variation in $G_{m}\left(\Delta G_{m}\right)$ can be given by using (54) and (56) as follows:

$$
\begin{aligned}
& \Delta G_{m} \\
& =\frac{k_{\text {in }}\left(k_{\text {in }} V_{\text {in }}+\left(k_{F}+k_{\mathrm{fd} 1}\right) V_{F}-\mathrm{V}_{\mathrm{TH} 1}\right)^{-1}}{\sqrt{\left(1+\lambda_{1} V_{F}\right)\left[1-\theta_{1}\left(k_{\mathrm{in}} V_{\mathrm{in}}+\left(k_{F}+k_{\mathrm{fd} 1}\right) V_{F}-\mathrm{V}_{\mathrm{TH} 1}\right)\right]}} \\
& \cdot \Delta I_{D 1},
\end{aligned}
$$




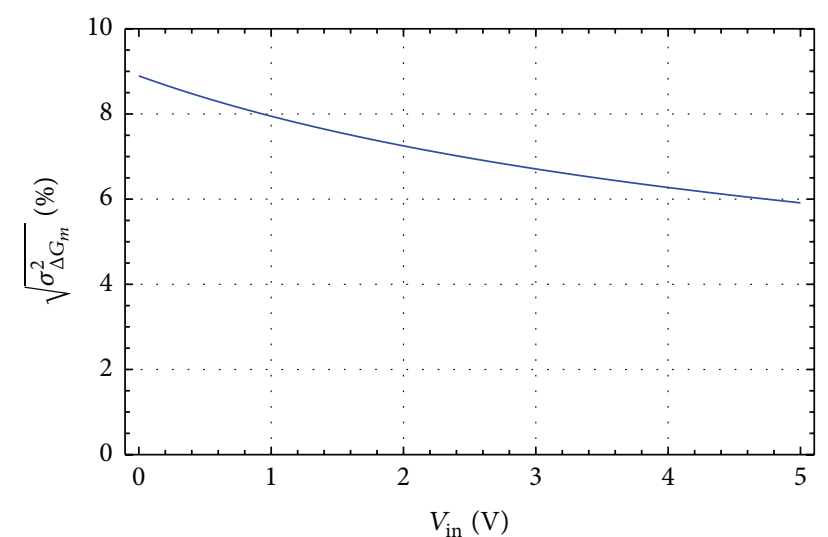

FIgURE 8: $\sqrt{\sigma_{\Delta G_{m}}^{2}}(\%)$ of the FGMOSFET based transconductor against $\left|V_{\text {in }}\right|$.

where $\Delta I_{D 1}$ denotes the process induced random variation in $I_{D 1}$ and can be determined by using (28)-(35) which belong to the saturation region part of the proposed model, with $N=$ 2 .

Moreover, it is necessary to model the statistical behavior of $\Delta G_{m}$ by using its variance $\left(\sigma_{\Delta G_{m}}^{2}\right)$ because its mean is equal to zero similarly to that of $\Delta I_{D 1}$. By using (55) and (56), $\sigma_{\Delta G_{m}}^{2}$ can be formulated as

$$
\begin{aligned}
& \sigma_{\Delta G_{m}}^{2} \\
& =\left[\frac{k_{\mathrm{in}}^{2}\left(k_{\mathrm{in}} V_{\mathrm{in}}+\left(k_{F}+k_{\mathrm{fd} 1}\right) V_{F}-\mathrm{V}_{\mathrm{TH} 1}\right)^{-2}}{\left(1+\lambda_{1} V_{F}\right)\left[1-\theta_{1}\left(k_{\mathrm{in}} V_{\mathrm{in}}+\left(k_{F}+k_{\mathrm{fd} 1}\right) V_{F}-\mathrm{V}_{\mathrm{TH} 1}\right)\right]}\right]^{2} \\
& \cdot \sigma_{\Delta I_{D 1}}^{2},
\end{aligned}
$$

where $\sigma_{\Delta I_{D 1}}^{2}$ denotes the variance of $\Delta I_{D 1}$ and can be analytically determined by using (29)-(36) which belong to the saturation region part proposed model, with $N=2$. It should be mentioned here that there is no correlation related term in (60) since $G_{m}$ is solely contributed by $I_{D 1}$. Finally, we can plot $\sqrt{\sigma_{\Delta G_{m}}^{2}}$ against $\left|V_{\text {in }}\right|$ as shown in Figure 8 where $\sqrt{\sigma_{\Delta G_{m}}^{2}}$ has been expressed as percentage of the nominal $G_{m}$.

\section{Conclusion}

In this research, the analytical model of $\Delta I_{D}$ of FGMOSFET has been proposed. The model is composed of two parts for triode and saturation regions of operation. The process induced device level random variations of each region, their statistical correlations, nonlinearity of $V_{\mathrm{FG}}$, and dependency on $V_{D}$ of the coupling factors have been taken into account. The proposed model has been found to be very accurate since it can fit the SPICE BSIM3v3 based reference obtained by using Monte-Carlo simulation and FGMOSFET simulation technique with SPICE with very high accuracy. If desired, it can also fit the BSIM4 based reference if the optimally extracted parameters obtained by using optimization algorithm have been applied.
By using the proposed model, the insight into the device level variability of FGMOSFET can be provided and the analytical model of process induced random variation in circuit level parameter of any FGMOSFET based circuit can be obtained. It has been shown that $\Delta I_{D}$ become more critical in low voltage/low power operating condition where the influences of $\Delta \beta, \Delta \theta$, and $\Delta V_{\mathrm{TH}}$ become relatively large compared to those of other variations. Furthermore, the effects of $\Delta \theta$ and $\Delta V_{\mathrm{TH}}$ can be reduced by lowering either $\theta$ or $V_{\mathrm{TH}}$ or both. So, this model has been found to be an efficient tool for the variability aware analysis/designing of any FGMOSFET based circuit.

\section{Conflict of Interests}

The author declares that there is no conflict of interests regarding the publication of this paper.

\section{Acknowledgment}

The author would like to acknowledge Mahidol University, Thailand, for the online database service.

\section{References}

[1] A. S. Medina-Vazquez, J. de la Cruz-Alejo, F. Gomez-Castañeda, and J. A. Moreno-Cadenas, "Low-voltage linear transconductor and a memory current using the MIFGMOS Transistor," International Journal of Electronics, vol. 96, no. 9, pp. 895-914, 2009.

[2] R. Pandey and M. Gupta, "FGMOS based voltage-controlled grounded resistor," Radioengineering, vol. 19, no. 3, pp. 455-459, 2010.

[3] M. Gupta and R. Pandey, "Low-voltage FGMOS based analog building blocks," Microelectronics Journal, vol. 42, no. 6, pp. 903-912, 2011.

[4] J. M. Miguel, A. J. Lopez-Martin, L. Acosta, J. Ramírez-Angulo, and R. G. Carvajal, "Using floating gate and quasi-floating gate techniques for rail-to-rail tunable CMOS transconductor design," IEEE Transactions on Circuits and Systems. I. Regular Papers, vol. 58, no. 7, pp. 1604-1614, 2011.

[5] A. J. Lopez-Martin, J. M. Algueta, C. Garcia-Alberdi, L. Acosta, R. G. Carvajal, and J. Ramirez-Angulo, "Design of micropower class AB transconductors: a systematic approach," Microelectronics Journal, vol. 44, no. 10, pp. 920-929, 2013.

[6] G.-C. Lizeth, T. Asai, and M. Motomura, "Application of nonlinear systems for designing low-power logic gates based on stochastic resonance," Nonlinear Theory and Its Applications, IEICE, vol. 5, no. 4, pp. 445-455, 2014.

[7] E. Rodriguez-Villegas, Low Power and Low Voltage Circuit Design with the FGMOS Transistor, vol. 20 of IET Circuits, Devices \& Systems Series, The Institution of Engineering and Technology, London, UK, 2006.

[8] V. S. Babu, R. S. Devi, A. Sekhar, and M. R. Baiju, "FGMOSFET circuit for neuron activation function and its derivative," in Proceedings of the 4th IEEE Conference on Industrial Electronics and Applications (ICIEA '09), pp. 739-744, IEEE, Xian, China, May 2009.

[9] M. Gupta, R. Srivastava, and U. Singh, "Low voltage floating gate MOS transistor based differential voltage squarer," ISRN Electronics, vol. 2014, Article ID 357184, 6 pages, 2014. 
[10] M. J. M. Pelgrom, A. C. J. Duinmaijer, and A. P. G. Welbers, "Matching properties of MOS transistors," IEEE Journal of SolidState Circuits, vol. 24, no. 5, pp. 1433-1440, 1989.

[11] S. K. Saha, "Modeling process variability in scaled CMOS technology," IEEE Design \& Test of Computers, vol. 27, no. 2, pp. 8-16, 2010.

[12] K. Kuhn, "Variability in nanoscale CMOS technology," Science China Information Sciences, vol. 54, no. 5, pp. 936-945, 2011.

[13] P. R. Kinget, "Device mismatch and tradeoffs in the design of analog circuits," IEEE Journal of Solid-State Circuits, vol. 40, no. 6, pp. 1212-1224, 2005.

[14] H. Masuda, T. Kida, and S.-I. Ohkawa, "Comprehensive matching characterization of analog CMOS circuits," IEICE Transactions on Fundamentals of Electronics, Communications and Computer Sciences, vol. 92, no. 4, pp. 966-975, 2009.

[15] F. Hong, B. Cheng, S. Roy, and D. Cumming, "An analytical mismatch model of nano-CMOS device under impact of intrinsic device variability," in Proceedings of the IEEE International Symposium of Circuits and Systems (ISCAS '11), pp. 2257-2260, May 2011.

[16] S. Vlassis and S. Siskos, "Design of voltage-mode and currentmode computational circuits using floating-gate MOS transistors," IEEE Transactions on Circuits and Systems I: Regular Papers, vol. 51, no. 2, pp. 329-341, 2004.

[17] A. E. Mourabit, P. Pittet, and G. N. Lu, "A low voltage, highly linear CMOS OTA," in Proceedings of the 16th International Conference on Microelectronics (ICM '04), pp. 700-703, tun, December 2004.

[18] Y. Zhai and P. A. Abshire, "Adaptive log domain filters for system identification using floating gate transistors," Analog Integrated Circuits and Signal Processing, vol. 56, no. 1-2, pp. 23-36, 2008.

[19] V. S. Babu, A. Sekhar, R. S. Devi, and M. R. Baiju, "Floating gate MOSFET based operational transconductance amplifier and study of mismatch," in Proceedings of the 4th IEEE Conference on Industrial Electronics and Applications (ICIEA '09), pp. 127132, IEEE, Xi'an, China, May 2009.

[20] A. E. Mourabit, G.-N. Lu, and P. Pittet, "Wide-linear-range subthreshold OTA for low-power, low-voltage, and low-frequency applications," IEEE Transactions on Circuits and Systems I: Regular Papers, vol. 52, no. 8, pp. 1481-1488, 2005.

[21] L.-L. Xiao, K. Liu, and D.-P. Han, "CMOS low data rate imaging method based on compressed sensing," Optics and Laser Technology, vol. 44, no. 5, pp. 1338-1345, 2012.

[22] S. K. Saha, "Non-linear coupling voltage of split-gate flash memory cells with additional top coupling gate," IET Circuits, Devices \& Systems, vol. 6, no. 3, pp. 204-210, 2012.

[23] J. Ramirez-Angulo, G. Gonzlrlez-Altamirano, and S. C. Choi, "Modeling multiple-input floating-gate transistors for analog signal processing," in Proceedings of the IEEE International Symposium on Circuits and Systems (ISCAS '97), vol. 3, pp. 20202023, IEEE, Hong Kong, June 1997.

[24] P. E. Allen and D. R. Holdberg, CMOS Analog Circuit Design, The Oxford Series in Electrical and Computer Engineering, Oxford University Press, Oxford, UK, 2011.

[25] W. Lü, G. Wang, and L. Sun, "Analytical modeling of cutoff frequency variability reserving correlations due to random dopant fluctuation in nanometer MOSFETs," Solid-State Electronics, vol. 105, pp. 63-69, 2015.

[26] K. Khu, "Statistical modeling for Monte Carlo simulation using Hspice," in Proceedings of the Synopsys Users Group Conference, pp. 1-10, 2006. 

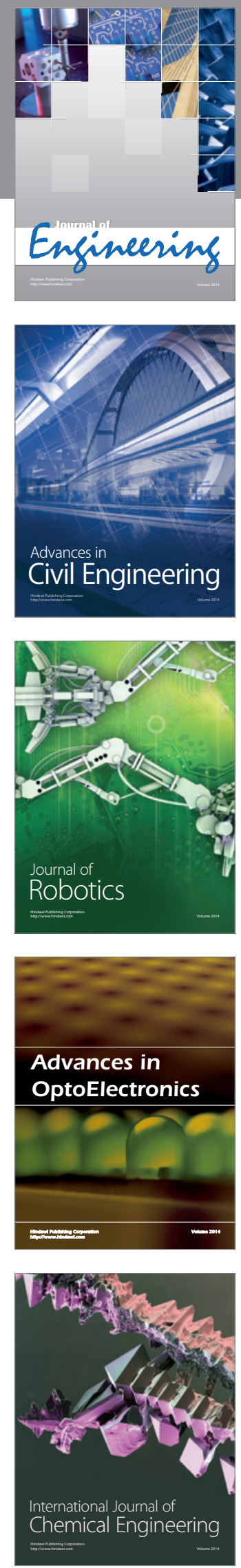

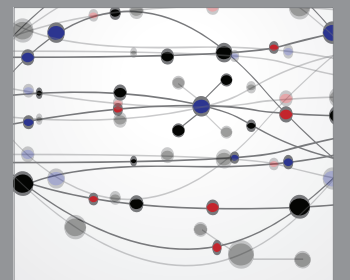

The Scientific World Journal
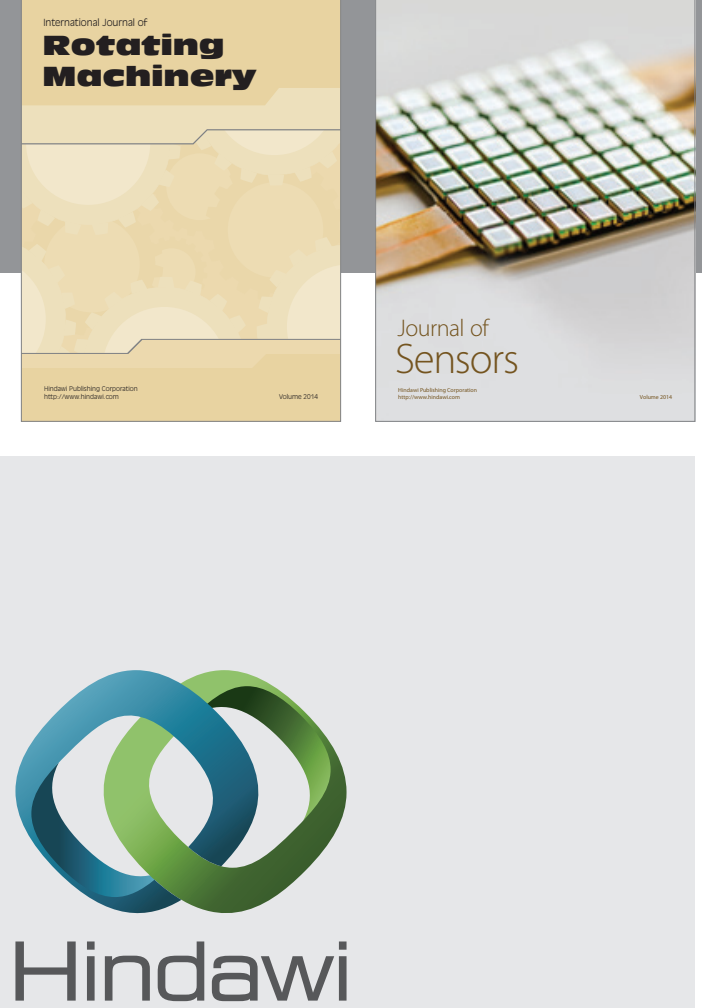

Submit your manuscripts at http://www.hindawi.com
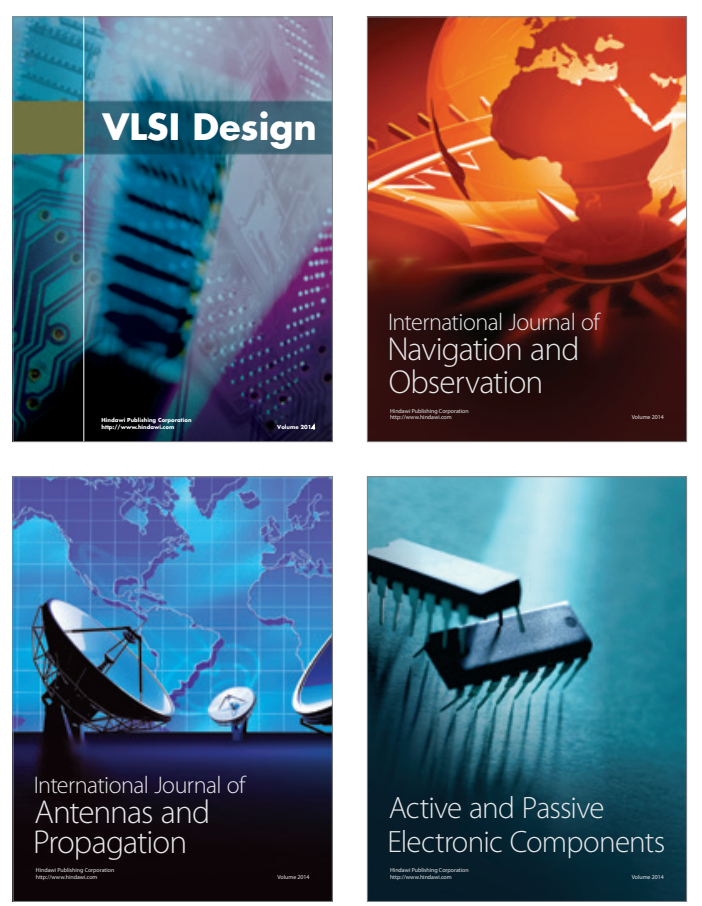
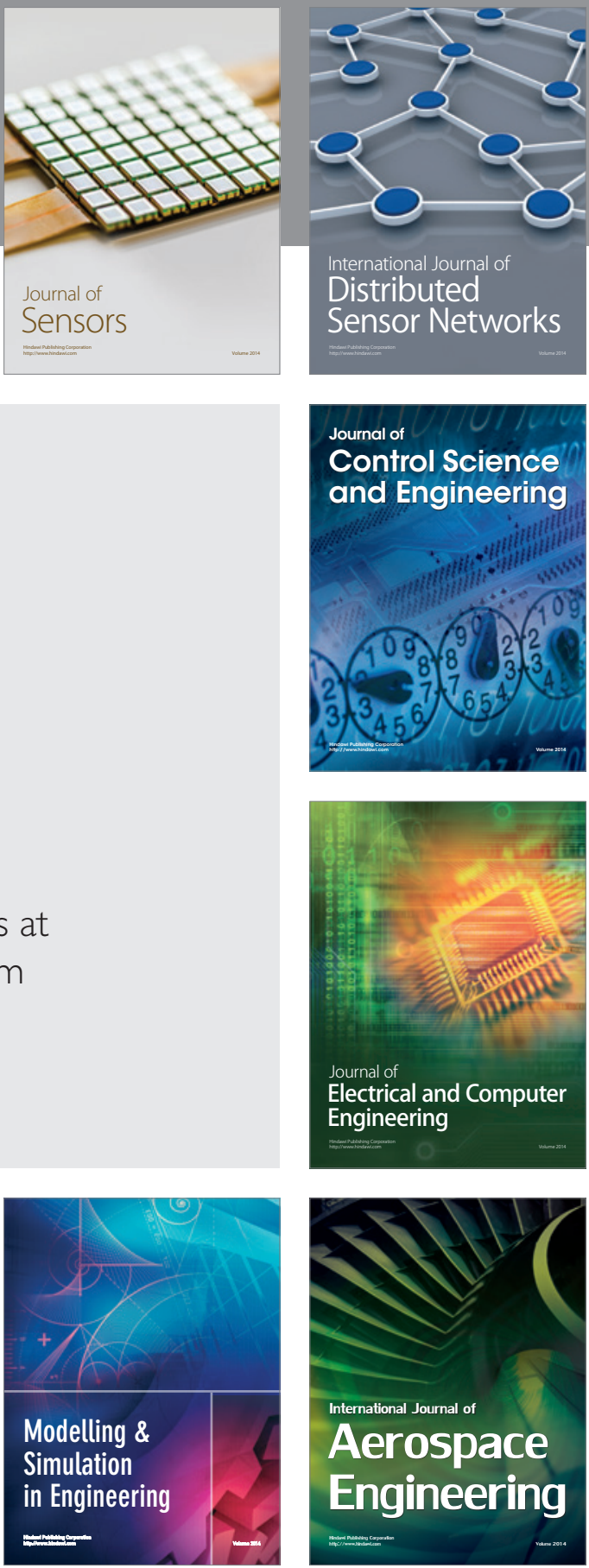

Journal of

Control Science

and Engineering
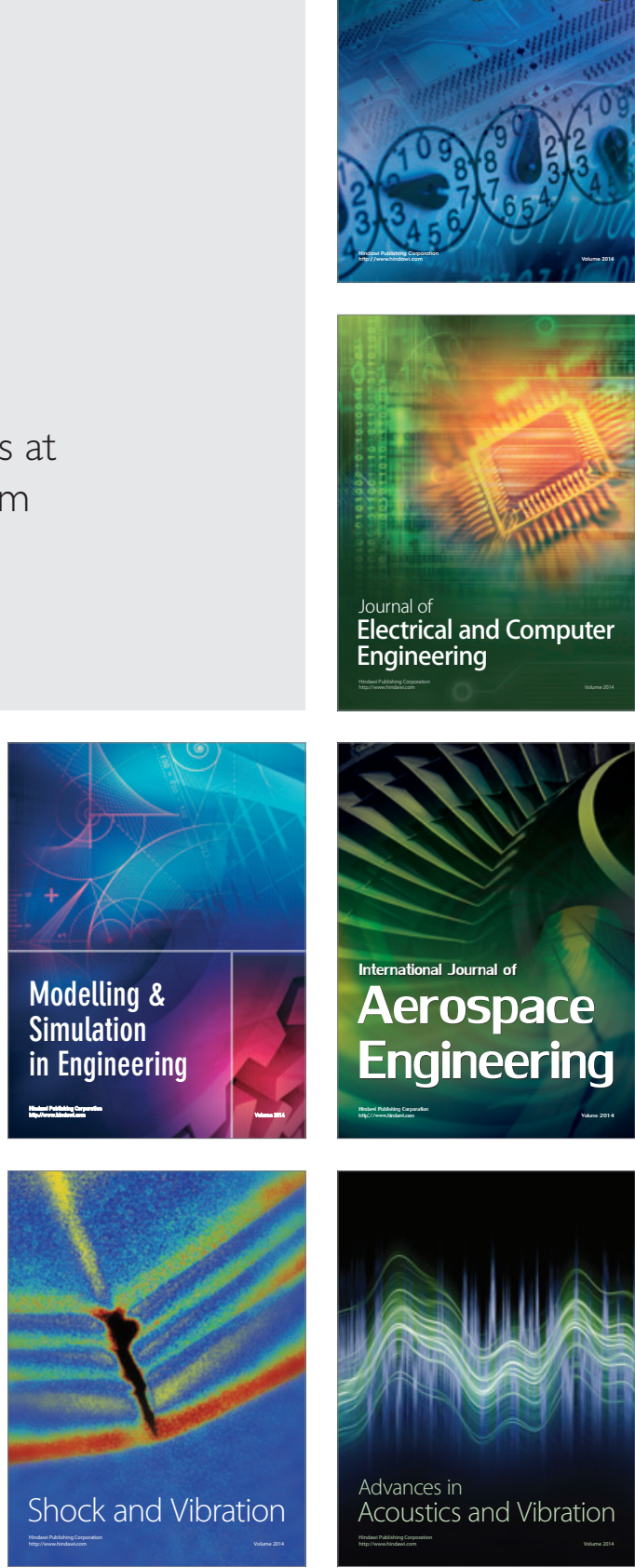